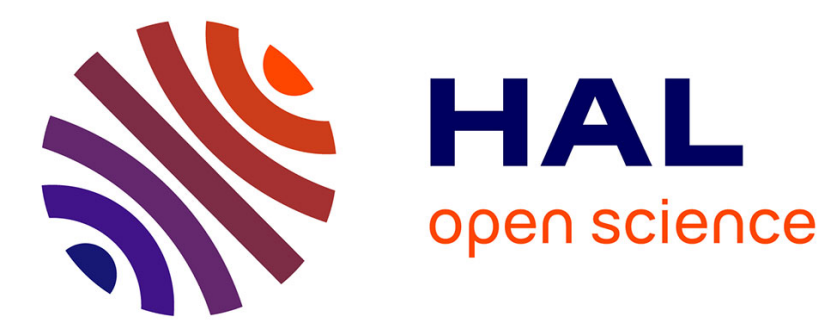

\title{
A 3D Reaction-Diffusion System Describing Bidomain Calcium Dynamics in Cardiac Cell
}

\author{
Mostafa Bendahmane, Elmahdi Erraji, Fahd Karami
}

\section{To cite this version:}

Mostafa Bendahmane, Elmahdi Erraji, Fahd Karami. A 3D Reaction-Diffusion System Describing Bidomain Calcium Dynamics in Cardiac Cell. Mathematical Modelling of Natural Phenomena, 2019, 14 (2), pp.27. 10.1051/mmnp/2018064 . hal-01680574

\section{HAL Id: hal-01680574 https://hal.inria.fr/hal-01680574}

Submitted on 30 Oct 2018

HAL is a multi-disciplinary open access archive for the deposit and dissemination of scientific research documents, whether they are published or not. The documents may come from teaching and research institutions in France or abroad, or from public or private research centers.
L'archive ouverte pluridisciplinaire HAL, est destinée au dépôt et à la diffusion de documents scientifiques de niveau recherche, publiés ou non, émanant des établissements d'enseignement et de recherche français ou étrangers, des laboratoires publics ou privés. 


\title{
A 3D REACTION-DIFFUSION SYSTEM DESCRIBING CALCIUM DYNAMICS IN CARDIAC CELL*
}

\author{
Mostafa Bendahmane ${ }^{1}$, Elmahdi Erraji ${ }^{2}$ and Fahd Karami ${ }^{3}$
}

\begin{abstract}
We are interested in modeling the interaction of calcium dynamics in a medium including sarcolemma and sarcoplasmic reticulum. The governing equations consist of a nonlinear reactiondiffusion system representing the various calcium fluxes and theirs buffers in the two media. We address the question of existence of weak solutions by using a fixed-point approach. We propose a finite element method for this system, we establish the existence of the discrete solution, and we show that the discrete solution generated by the given scheme converges to the corresponding weak solution for the model studied. Finally, we give some 2D and 3D numerical examples to our model.
\end{abstract}

Mathematics Subject Classification. 92C50, 65M60, 65N12, 92C40.

The dates will be set by the publisher.

\section{Contents}

Introduction

1. Calcium transmission model 3

1.1. Description of calcium transmission dynamics 4

1.2. Description of buffers dynamics 4

1.3. Boundary condition 5

2. Well-posedness (existence and uniqueness) of the weak solution: Continuous case. 7

2.1. Existence result to the regularized problem: the fixed-point method 8

2.2. Existence of weak solutions 12

3. Numerical approximation 13

3.1. The semi-discrete scheme 13

3.2. Numerical convergence analysis 14

4. Numerical simulation 19

Keywords and phrases. Finite element, Calcium dynamics, Anomalous diffusion, Convergence, Interface problem, Medical modeling.

* MB would like to thank the Fincome Moroccan research project for supporting this work.

${ }^{1}$ Institut de mathématique de Bordeaux (IMB) et l'institut de rythmologie et modélisation cardiaque (Liryc), université de Bordeaux et INRIA-Carmen Bordeaux Sud-Ouest; e-mail: mostafa.bendahmane@u-bordeaux.fr

${ }^{2}$ Ecole Supérieure de Technologie d'Essaouira, Université Cadi Ayyad, B.P. 383 Essaouira El Jadida, Essaouira, Morocco, Morocco; e-mail: erraji0elmahdi@gmail.com

3 Ecole Supérieure de Technologie d'Essaouira, Université Cadi Ayyad, B.P. 383 Essaouira El Jadida, Essaouira, Morocco, Morocco; e-mail: fa.karami@uca.ma 
2D numerical simulation of calcium model

3D numerical simulation of calcium model $\quad 22$

Conclusion \& Perspective $\quad 25$

\begin{tabular}{l} 
References \\
\hline
\end{tabular}

\section{INTRODUCTION}

The study of calcium dynamics in cardiac cell is a priority to reveal the mysterious causes of cell failure. One of the principal causes of heart failure is the perturbation of calcium induced-calcium release (CICR) cycle of a cardiac cell (see for e.g. [20]). The calcium cycle in cardiac cell is a complicated process, since it involves different mechanisms such as swapping between the cytoplasm and sarcoplasmic reticulum through different type of channels and interactions with biochemical species in the cell medium. On the cellular scale, the perturbation of CICR could be explained by pathological behavior of ionic channels especially Ryanodine receptor (RyR). The scientific community is convinced, based on strong evidence (we refer to [33]) that a vast majority of pathological heart cases is due to RyR irregularity. Which induces more questions than answers about understanding the causes and designing an effective therapeutic strategy on the cellular scale. This would require a larger collaboration between various scientific fields including physics, chemistry, biology and mathematics.

In cardiac and other muscle cells, calcium signaling is the main factor to mediate excitation-contraction coupling (ECC). Its cycle lies between the action potential and muscle contraction. The ECC is a consequence of a propagating action potential through muscle cell membranes. At the cellular scale, action potential propagates along the cell membrane including the T-tubules which forms a tubular cavity inside the cell. This action potential triggered voltage opened a channel (VOC) or L-type channels located on the cell membrane surface. As a result, a passive flux of $\mathrm{Ca}^{2+}$ crosses the L-type due to the large difference of calcium concentration between the extracellular $\left(\left[\mathrm{Ca}^{2+}\right]_{e} \approx 1 m M\right)$ and the intracellular $\left(\left[\mathrm{Ca}^{2+}\right]_{i} \approx 0.1 \mu M\right)$ medium. The rising $\mathrm{Ca}^{2+}$ concentration triggered the calcium-induced calcium release process (CICR). It begins by triggering a ryanodine receptor located on sarcoplasmic reticulum, this allows the $\mathrm{Ca}^{2+}$ influx into intracellular medium from sarcoplasmic reticulum, where $C a^{2+}$ is stored at elevated concentration $\left(\left[\mathrm{Ca}^{2+}\right]_{s r} \approx 1.3 \mathrm{mM}\right.$, see $[2]$ for this detail), not to mention the calcium concentration maintained by the heavy buffering. This leads to the simultaneous opening of the other ionic channel. Then, the influx of $\mathrm{Ca}^{2+}$ ion diffuses through myofibrils to cause mechanical activity.

In literature, many authors attempt to build a primal model of calcium dynamics. We mention, for example the works of Peskoff et al. in [17,25], their first work establishes a primal model of calcium distribution evolution in diad neighbor. A more complete calcium model is established by extending the previous model to the whole cell and considering interaction with local buffers in the medium (see [26] for more details). In passing, we want to mention the work of Sobie et al. in [31], where the authors studied the dynamics of $\mathrm{Ca}^{2+}$ under new consideration on RyR Stochastic behavior, period of release and there distribution over space. Note that these studies of spatial calcium dynamics doesn't take in consideration the complexity of cell medium (for example the presence of mitochondria in the cell and the heterogenerous of the media). This will affect the calcium diffusion and produce some serious needs in the modeling part. Recently, some new spatial calcium models are based on a transmission defined by interaction in a local position of ionic channels (see for e.g. [5, 17]). These models can be interpreted as a transmission boundary value problem and can be studied by using domain decomposition techniques. From the analytical and the numerical perspectives, there are many results that has been established in this context (see for e.g. [9, 16, 21]).

Note that the previous spatial calcium models are governed by a linear diffusion. We know that the solution of a linear diffusion equation admits an infinite speed of propagation whereas the solution of the nonlinear diffusion equation shows a finite speed of propagation. Moreover, from the application point of view it is not realistic to have the property of infinite speed of propagation because the calcium can not diffuse infinitely. In this paper, 
we establish the mathematical model of species dynamics in cardio-myocyte with nonlinear diffusion. Note that strong experimental evidence proves the complexity of calcium diffusion (see for e.g. [8]). Theoretically, the ionic nature of $\mathrm{Ca}^{2+}$ as well as the complexity of the cytosolic and sacroplasmic medium imposes some modifications on the previous models (we refer here to the works $[27,28]$ ). Moreover, advanced microscopical imaging technique shows that the cytoplasm of mammalian cardiac myocytes seems to be highly heterogeneous and anisotropic due to the dense presence of some organelles. We mention, for example the presence of mitochondria and Golgi complex on the micron-scale. A zoom into a smaller scale, clarify the presence of a network of elements such as microtubule and actin filaments. Hence, based on strong experimental evidence shown in [10], the authors highly recommend to consider an anomalous diffusion in the cell medium. Based on the same arguments between others [29], a recent work [12] has considered a porous medium model describing cardiac-electro-physiology.

Our main contribution in this context is to generalize the previous models to a nonlinear model with non constant diffusion coefficients. This paper is devoted to a rigorous study of existence and uniqueness of $L^{\infty}$ bounded solutions of the proposed model. Moreover, we establish a fully discrete scheme in finite element framework for spatial discretization, whereas the first order backward Euler scheme is used for time discretization. Then, we move forward to prove the convergence result of the proposed scheme. We want to mention here (in the context of finite element method) that we cannot obtain the maximum principle as in the continuous case because the positive and negative cuts test functions may not be admissible. For the convergence of the scheme, we will assume that the initial conditions are only bounded in $L^{2}$.

The plan of this paper is as follows: In Section 1 we propose the derivation of the calcium model based on conservation law and interaction between species. Section 2 is devoted to the existence and uniqueness of weak solution (defined in Definition 2.1 below) by using the Schauder fixed point theorem and the passage to the limit that transforms the regularized system into the original problem. We introduce in Section 3 some notations for the finite element method and we present our scheme. The proof of the convergence result (of the scheme) is divided into a priori estimates, compactness for discrete solution and convergence to a weak solution. Finally, we give in Section 4 some numerical examples to our model.

\section{Calcium transmission model}

We devote this section to a brief derivation of the calcium model of cardiac tissue. As principal references for this model with constant diffusion we found [5]. Before starting our calcium dynamics modeling, we refer to Figure 1 for a good description of the cytosolic and sarcoplasmic reticulum domains.

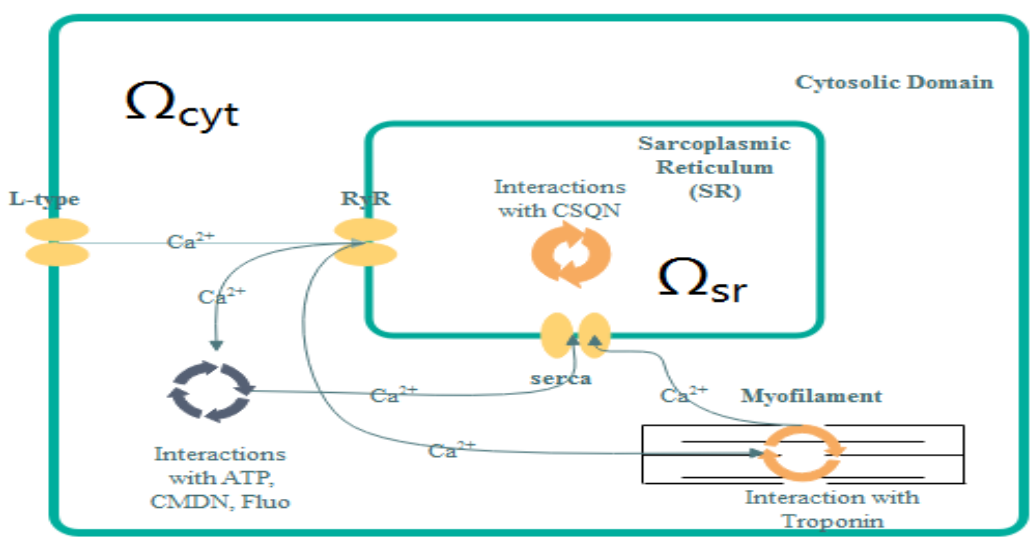

Figure 1. Description of calcium cycle in Cardiac cell 


\subsection{Description of calcium transmission dynamics}

In both sub-domains, calcium behaves in a similar manner. To derive the partial differential equation controlling calcium behavior, we consider an arbitrary domain $\Omega$. By the conservation law, we have

$$
\frac{d}{d t} \int_{\Omega} c d V=\int_{\Omega} R d V-\int_{\partial \Omega} J \cdot \eta d A,
$$

where $c$ is the calcium concentration, $J$ its flux trough $\partial \Omega, R$ its source inside $\Omega$ due to interaction of $C a^{2+}$ with other species and $\eta$ is the normal vector on $\partial \Omega$.

Now to conclude this equation, we use Stokes Ostradski formula

$$
\int_{\partial \Omega} J \cdot \eta d A=\int_{\Omega} \operatorname{div} J d V
$$

Combining (1) and (2), we arrive

$$
\frac{d}{d t} \int_{\Omega} c d V=\int_{\Omega} R d V-\int_{\Omega} \operatorname{div} J \cdot \eta d V .
$$

This implies

$$
\partial_{t} c=R-\operatorname{div} J \text { in }(0, T) \times \Omega .
$$

Thanks to Fick law, the calcium moves from high concentration to low concentration in cell medium. So the flux $J$ is directed in the opposite direction as the concentration gradient. Moreover, the diffusion coefficient depend on solvent concentration. In summary, calcium flux can be written as

$$
J=-D_{c}(c) \nabla c
$$

where $D_{c}$ is a diffusion-dependent function of $c$. Hence, we deduce :

$$
\partial_{t} c=\operatorname{div}\left(D_{c}(c) \nabla c\right)+R_{c} \text { in }(0, T) \times \Omega,
$$

and similarly the equation of $C a^{2+}$ in $\mathrm{SR}$ is:

$$
\partial_{t} s=\operatorname{div}\left(D_{s}(s) \nabla s\right)+R_{s} \text { in }(0, T) \times \Omega,
$$

where $D_{s}$ is a diffusion-dependent function of $s$. We will define the sources $R_{c}$ and $R_{s}$ explicitly in the following sub-sections.

\subsection{Description of buffers dynamics}

In this subsection, we establish the equations governing by the buffers (recall that the buffers are proteins that interact with calcium to produce another molecule). We assume that the total concentration of the free buffer [buffer] and the new buffer [buffer- $\mathrm{Ca}^{2+}$ ] (resulting from the interaction of the free buffer with $\mathrm{Ca}^{2+}$ ) is a constant in time. The chemical equation describing this phenomenon is:

$$
\text { Buffer }+C a^{2+} \underset{k_{o f f}}{\stackrel{k_{o n}}{\rightleftharpoons}} \text { Buffer- } C a^{2+}
$$

where $k_{o f f}$ and $k_{o n}$ are two rates coefficients.

Next we let $b$ the concentration of buffer- $C a^{2+}, B_{t o t}$ the total concentration of the buffer and $B$ the concentration of buffer only. An application of the law of mass action (see for e.g [14]), we obtain

$$
\partial_{t} b=R(c, b)=k_{o n} c B-k_{o f f} b .
$$


We notice that $B=B_{t o t}-b$ so the equation becomes:

$$
\partial_{t} b=R(c, b)=k_{o n} c\left(B_{t o t}-b\right)-k_{o f f} b .
$$

Each buffer (for more information, check [23]) is described by a conservation law similar to (1). So we conclude a similar PDE. The buffer list living in the cytosol is :

- ATP or nucleoside triphosphate used in the cells as a coenzyme often called the molecular currency of intracellular energy transfer.

$$
\partial_{t} b_{1}=D_{b_{1}} \Delta b_{1}+R_{1}\left(c, b_{1}\right),
$$

where $D_{b_{1}}$ is a diffusion coefficient and the source term $R_{1}\left(c, b_{1}\right):=k_{1}^{o n} c\left(B_{1}-b_{1}\right)-k^{o f f} b_{1}$.

- $\mathrm{CMDN}$ or calmodulin is a multi-functional intermediate calcium-binding messenger protein.

$$
\partial_{t} b_{2}=D_{b_{2}} \Delta b_{2}+R_{2}\left(c, b_{2}\right),
$$

where $D_{b_{2}}$ is a diffusion coefficient and the source term $R_{2}\left(c, b_{2}\right):=k_{2}^{o n} c\left(B_{2}-b_{2}\right)-k^{o f f} b_{2}$.

- Fluo-4 is used in the life sciences generally as a non destructive way of tracking or analyzing biological molecules such as $\mathrm{Ca}^{2+}$.

$$
\partial_{t} b_{3}=D_{b_{3}} \Delta b_{3}+R_{3}\left(c, b_{3}\right)
$$

where $D_{b_{3}}$ is a diffusion coefficient and the source term $R_{3}\left(c, b_{3}\right)=k_{3}^{o n} c\left(B_{3}-b_{3}\right)-k^{o f f} b_{3}$.

- TRPN or troponin is a protein of muscle that together with tropomyosin forms a regulatory protein complex controlling the interaction of actin and myosin and when combined with calcium ions permits muscular contraction. Similarly to Fluo-4, the protein TRPN satisfies the following equation

$$
\partial_{t} b_{4}=D_{b_{4}} \Delta b_{4}+R_{4}\left(c, b_{4}\right),
$$

where $D_{b_{4}}$ is a diffusion coefficient and the source term $R_{4}\left(c, b_{4}\right)=k_{4}^{o n} c\left(B_{4}-b_{4}\right)-k^{o f f} b_{4}$.

We consider only one buffer living in SR :

- CSQN or calsequestrin is a calcium binding protein. It helps hold calcium in the citerna of the sarcoplasmic reticulum after a muscle contraction. Moreover, the protein CSQN is governed by the equation

$$
\partial_{t} b_{5}=D_{b_{5}} \Delta b_{5}+R_{5}\left(s, b_{5}\right),
$$

where $D_{b_{5}}$ is a diffusion coefficient and the source term $R_{5}\left(s, b_{5}\right)=k_{5}^{o n} s\left(B_{5}-b_{5}\right)-k^{o f f} b_{5}$.

The interaction between buffers and calcium define the source terms $R_{c}$ and $R_{s}$ mentioned in (6) and (7).

$$
R_{c}=-\sum_{i=1}^{4} R_{i}, \quad R_{s}=-R_{5} .
$$

\subsection{Boundary condition}

In cardiac cell's, the calcium concentration in SR is considerably high. The activation of ionic channels and $\mathrm{Ca}^{2+}$ low concentration in cytosol induces a calcium flow throw RyR proportional to its difference between the two sub-domains:

Due to Ficks law, we have

$$
J \cdot \eta= \pm g(s-c) .
$$

$$
D_{s}(s) \nabla s \cdot \eta_{s}=g(s-c), \text { on } \Gamma_{r y r, T}:=\Gamma_{r y r} \times(0, T),
$$

and

$$
D_{c}(c) \nabla c \cdot \eta_{c}=-g(s-c) \text { on } \Gamma_{r y r, T}:=\Gamma_{r y r} \times(0, T),
$$




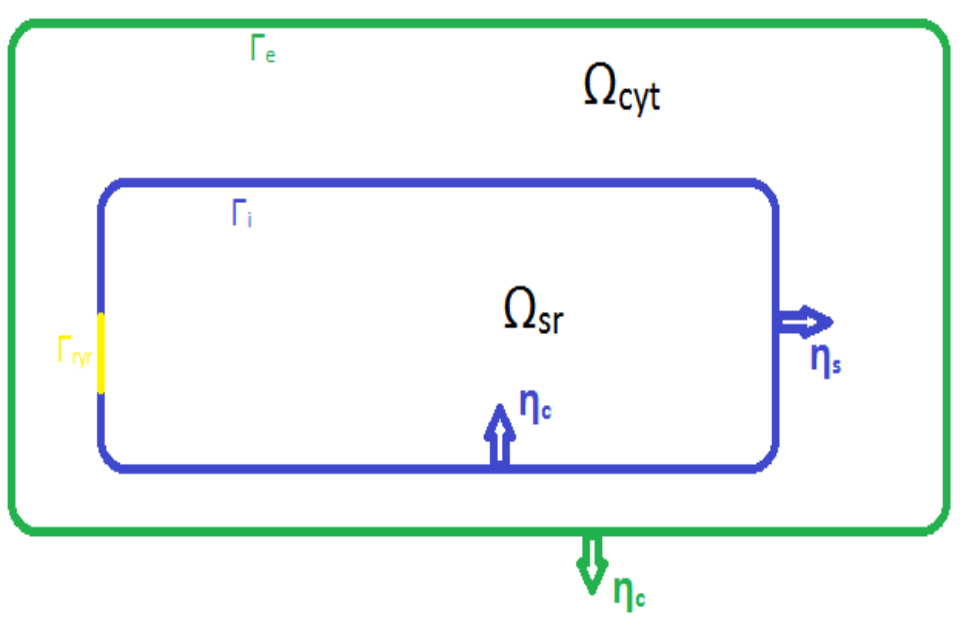

FiguRE 2. A Graphical representations of the boundary of the calcium model. We note the sarcoplasmic membrane $\Gamma^{s r}=\Gamma_{i} \cup \Gamma_{r y r}$ and cytosolic membrane $\Gamma=\Gamma_{e} \cup \Gamma_{i} \cup \Gamma_{r y r}$, while we consider $\Gamma_{i s o}^{c y t}=\Gamma_{e} \cup \Gamma_{i}$ the impermeable part of the cytosolic membrane, $\Gamma_{i s o}^{s r}=\Gamma_{i}$ is the impermeable part of the sarcoplasmic membrane.

where $\eta_{c}\left(\right.$ Resp. $\left.\eta_{s}\right)$ is the outward normal vector with respect to $\Omega_{c y t}\left(\right.$ Resp. $\left.\Omega_{s r}\right), \Gamma_{r y r}$ is subset of $\partial \Omega_{s r} \cup \partial \Omega_{c y t}$ and $\Gamma_{r y r, T}:=\Gamma_{r y r} \times(0, T)$.

Since the SR and cell membrane are impermeable. Then, there is no flux through it. This can be implemented as an homogeneous Neumann boundary condition

$$
D_{s}(s) \nabla s \cdot \eta_{s}=0 \text { on } \Gamma_{i s o, T}^{c y t}:=\Gamma_{i s o}^{c y t} \times(0, T),
$$

and

$$
D_{c}(c) \nabla c \cdot \eta_{c}=0 \text { on } \Gamma_{i s o, T}^{s r}:=\Gamma_{i s o}^{s r} \times(0, T),
$$

where $\Gamma_{i s o}^{i}:=\partial \Omega_{i}-\Gamma_{\text {ryr }}$ for $i=c y t, s r$.

The flux of buffers is null through cell membrane

$$
\nabla b_{i} \cdot \eta_{c}=0, \text { on } \Gamma_{T}:=\Gamma \times(0, T), \quad \text { for } i=1,2,3,4 \text {, }
$$

and

$$
\nabla b_{5} \cdot \eta_{s}=0 \text { on } \Gamma_{T}^{s r}:=\Gamma^{s r} \times(0, T) .
$$


Finally, combining the previous equations, we get the following nonlinear reaction-diffusion system

$$
\begin{cases}\partial_{t} c=\operatorname{div}\left(D_{c}(c) \nabla c\right)-\sum_{i=1}^{4} R_{i}\left(c, b_{i}\right) & \text { in } \Omega_{c y t, T}:=\Omega_{c y t} \times(0, T), \\ \partial_{t} b_{i}=D_{b_{i}} \Delta b_{i}+R_{i}\left(c, b_{i}\right) & \text { in } \Omega_{c y t, T} \text { for } i=1, \ldots, 4, \\ \partial_{t} s=\operatorname{div}\left(D_{s}(s) \nabla s\right)-R_{s}\left(s, b_{5}\right) & \text { in } \Omega_{s r, T}:=\Omega_{s r} \times(0, T), \\ \partial_{t} b_{5}=D_{b_{5}} \Delta b_{5}+R_{5}\left(s, b_{5}\right) & \text { in } \Omega_{s r, T}, \\ D_{c}(c) \nabla c \cdot \eta_{c}=0 & \text { on } \Gamma_{i s o, T}^{c y t}:=\Gamma_{i s o}^{c y t} \times(0, T), \\ D_{s}(s) \nabla s \cdot \eta_{s}=0 & \text { on } \Gamma_{i s o, T}^{s r}:=\Gamma_{i s o}^{s r} \times(0, T), \\ \nabla b_{i} \cdot \eta_{c}=0 & \text { on } \Gamma_{T}:=\Gamma \times(0, T), i=1, \ldots, 4, \\ \nabla b_{5} \cdot \eta_{c}=0 & \text { on } \Gamma_{T}^{s r}:=\left(\Gamma_{i} \cup \Gamma_{r y r}\right) \times(0, T), \\ D_{s}(s) \nabla s \cdot \eta_{s}=-g(s-c) & \text { on } \Gamma_{r y r, T}:=\Gamma_{r y r} \times(0, T), \\ D_{c}(c) \nabla c \cdot \eta_{c}=g(s-c) & \text { on } \Gamma_{r y r, T}:=\Gamma_{r y r} \times(0, T), \\ \left(c, b_{i}\right)(\cdot, 0)=\left(c_{0}, b_{i, 0}\right)(\cdot) \text { in } \Omega_{c y t} \text { and } & \left(s, b_{5}\right)(\cdot, 0)=\left(s_{0}, b_{5,0}\right)(\cdot) \text { in } \Omega_{s r} .\end{cases}
$$

In this system, the nonlinear diffusivities $D_{c}, D_{s}$ are assumed continuous, positive, coercive, and there exist constants $0<D^{\min }<D^{\max }$ :

$D_{s}, D_{c}$ continuous, $0<D^{\min } \leq D_{s}(m), D_{c}(m) \leq D^{\max }<\infty$ for $m \in \mathbb{R}$, and $0<D^{\min } \leq D_{b_{i}}$ where $D_{b_{i}}$ is a constant diffusion for $i=1, \ldots, 4$.

Remark 1. Because of the membrane impermeability, we considered in our model the Neumann boundary condition on buffers equations. For calcium dynamics in both sub-domain, the cell membrane contains the ionic channel RyR that allows only the transmission of $\mathrm{Ca}^{2+}$. Hence, we have restricted the transmission boundary condition only for calcium equations on $\Gamma_{r y r}$. Moreover, we want to mention that the initial data $b_{i, 0}$ of the buffer should be less than its total concentration $B_{i}$ (Total concentration with or without calcium) for $i=1, \ldots, 5$. In the paper [4], the author has studied the diffusivity of some buffers (In our case $b_{4}$ and $\left.b_{5}\right)$. Without loss of generality, in this work we consider the small diffusion coefficient in troponin $\left(b_{4}\right)$ and calsequestrin $\left(b_{5}\right)$ equations.

\section{WELL-POSEDNESS (EXISTENCE AND UNIQUENESS) OF THE WEAK SOLUTION: Continuous CASE.}

In this section, we introduce the variational formulation of the problem (16). Then, we look after existence results.

Definition 2.1 ( $L^{2}$ bound solution). A weak $L^{2}$ bound solution of (16) is a seven component $\left(c, b_{1}, b_{2}, b_{3}, b_{4}, s\right.$, $\left.b_{5}\right)$ such that $c, b_{i} \in L^{\infty}\left(0, T ; L^{2}\left(\Omega_{c y t}\right)\right) \cap L^{2}\left(0, T ; H^{1}\left(\Omega_{c y t}\right)\right)$ and $s, b_{5} \in L^{\infty}\left(0, T ; L^{2}\left(\Omega_{s r}\right)\right) \cap L^{2}\left(0, T ; H^{1}\left(\Omega_{s r}\right)\right)$, 
$\partial_{t} c, \partial_{t} b_{i} \in L^{2}\left(0, T ; H^{1}\left(\Omega_{c y t}\right)^{\prime}\right), \partial_{t} s, \partial_{t} b_{5} \in L^{2}\left(0, T ; H^{1}\left(\Omega_{s r}\right)^{\prime}\right)$, and satisfying

$$
\begin{aligned}
& \int_{0}^{T}\left\langle\partial_{t} c, \varphi_{c}\right\rangle_{c y t} d t+\iint_{\Omega_{c y t, T}} D_{c}(c) \nabla c \cdot \nabla \varphi_{c} d x d t-\iint_{\Gamma_{r y r}, T} g(s-c) \varphi_{c} d \sigma d t \\
& =-\iint_{\Omega_{c y t, T}} \sum_{i=1}^{4} R_{i}\left(c, b_{i}\right) \varphi_{c} d x d t \\
& \int_{0}^{T}\left\langle\partial_{t} s, \varphi_{s}\right\rangle_{s r} d t+\iint_{\Omega_{s r, T}} D_{s}(s) \nabla s \cdot \nabla \varphi_{s} d x d t+\iint_{\Gamma_{r y r}, T} g(s-c) \varphi_{s} d \sigma d t \\
& =-\iint_{\Omega_{s r, T}} R_{5}\left(s, b_{5}\right) \varphi_{s} d x d t, \\
& \int_{0}^{T}\left\langle\partial_{t} b_{i}, \varphi_{i}\right\rangle_{c y t} d t+D_{b_{i}} \iint_{\Omega_{c y t, T}} \nabla b_{i} \cdot \nabla \varphi_{i} d x d t=\iint_{\Omega_{c y t, T}} R_{i}\left(c, b_{i}\right) \varphi_{i} d x d t \\
& \int_{0}^{T}\left\langle\partial_{t} b_{5}, \varphi_{5}\right\rangle_{s r} d t+D_{b_{5}} \iint_{\Omega_{c y t, T}} \nabla b_{5} \cdot \nabla \varphi_{5} d x d t=\iint_{\Omega_{s r, T}} R_{5}\left(c, b_{5}\right) \varphi_{5} d x d t
\end{aligned}
$$

for all $\varphi_{c}, \varphi_{i} \in L^{2}\left(0, T ; H^{1}\left(\Omega_{c y t}\right)\right), \varphi_{s}, \varphi_{5} \in L^{2}\left(0, T ; H^{1}\left(\Omega_{s r}\right)\right)$ for $i=1, \ldots, 4$. Here, $\langle\cdot, \cdot\rangle$ denotes the duality pairing between $H^{1}$ and $\left(H^{1}\right)^{\prime}$

Definition 2.2 ( $L^{\infty}$ bound solution). A weak $L^{\infty}$ bound solution of (16) is a nonnegative and a $L^{\infty}$ function with seven components $\left(c, b_{1}, b_{2}, b_{3}, b_{4}, s, b_{5}\right)$ satisfying Definition 2.1.

Our first main result is the following existence theorem for weak solutions.

Theorem 2.3. Assume that condition (17) holds. If $c_{0}, b_{1,0}, b_{2,0}, b_{3,0}, b_{4,0} \in L^{\infty}\left(\Omega_{c y t}\right)$ and $s_{0}, b_{5,0} \in L^{\infty}\left(\Omega_{s r}\right)$, then the system (16) possesses a unique weak solution in sense of Definition 2.2.

To prove theorem 2.3 we first prove existence of solutions to the approximate problem (19) below by applying the Schauder fixed-point theorem (in an appropriate functional setting). Then we send the regularization parameter $\epsilon$ to zero to produce a weak solution of the original system (16) as the limit of a sequence of such approximate solutions. Convergence is achieved by means of a priori estimates and compactness arguments.

\subsection{Existence result to the regularized problem: the fixed-point method}

In this subsection we prove, for each fixed $\varepsilon>0$, the existence of solutions to the approximate problem (19) below, by applying the Schauder fixed-point theorem. For this purpose, we introduce the following closed subset of the Banach space

$$
\mathcal{A}=\left\{(c, s) \in H: 0 \leq c\left(t, x_{c}\right), s\left(t, x_{s}\right) \leq R \text { for a.e. }\left(t, x_{c}\right) \in \Omega_{c y t, T} \text { and }\left(t, x_{s}\right) \in \Omega_{s r, T}\right\},
$$


for some constant $R>0$. Herein, $H:=L^{2}\left(\Omega_{c y t, T}\right) \times L^{2}\left(\Omega_{s r, T}\right)$. For a given nonnegative function $\hat{U}=(\hat{c}, \hat{s})$ such that $\hat{c} \in L^{2}\left(\Omega_{c y t, T}\right)$ and $\hat{s} \in L^{2}\left(\Omega_{s r, T}\right)$, we solve the approximate system

$$
\begin{cases}\partial_{t} c=\operatorname{div}\left(D_{c}(\hat{c}) \nabla c\right)-\sum_{i=1}^{4} R_{i, \varepsilon}\left(c^{+}, b_{i}^{+}\right) & \text {in } \Omega_{c y t, T}, \\ \partial_{t} b_{i}=D_{b_{i}} \Delta b_{i}+R_{i, \varepsilon}\left(\hat{c}^{+}, b_{i}^{+}\right) & \text {in } \Omega_{c y t, T}, \text { for } i=1, \ldots, 4, \\ \partial_{t} s=\operatorname{div}\left(D_{s}(\hat{s}) \nabla s\right)-R_{s, \varepsilon}\left(s^{+}, b_{5}^{+}\right) & \text {in } \Omega_{s r, T}, \\ \partial_{t} b_{5}=D_{b_{5}} \Delta b_{5}+R_{5, \varepsilon}\left(\hat{s}^{+}, b_{5}^{+}\right) & \text {in } \Omega_{s r, T}, \\ D_{c}(c) \nabla c \cdot \eta_{c}=0 & \text { on } \Gamma_{i s o, T}^{c y t}, \\ D_{s}(s) \nabla s \cdot \eta_{s}=0 & \text { on } \Gamma_{i s o, T}^{s r}, \\ \nabla b_{i} \cdot \eta_{c}=0 & \text { on } \Gamma_{T}, \text { for } i=1, \ldots, 4 \\ \nabla b_{5} \cdot \eta_{s}=0 & \text { on } \Gamma_{T}^{s r}, \\ D_{s}(s) \nabla s \cdot \eta_{s}=-g(s-c) & \text { on } \Gamma_{r y r, T}, \\ D_{c}(c) \nabla c \cdot \eta_{c}=g(s-c) & \text { on } \Gamma_{r y r, T}, \\ \left(c, b_{i}\right)(\cdot, 0)=\left(c_{0}, b_{i, 0}\right)(\cdot) \text { in } \Omega_{c y t} \text { and } & \left(s, b_{5}\right)(\cdot, 0)=\left(s_{0}, b_{5,0}\right)(\cdot) \text { in } \Omega_{s r},\end{cases}
$$

defined for each $\varepsilon>0$. Herein,

$$
R_{\kappa, \varepsilon}=\frac{R_{\kappa}}{1+\varepsilon\left|R_{\kappa}\right|} \text { for } \kappa=1, \ldots, 5 .
$$

Next, we introduce the map $\mathcal{F}$ defined by

$$
\begin{aligned}
\mathcal{F}: \mathcal{A} & \longmapsto \mathcal{A} \\
\hat{U} & \longrightarrow U,
\end{aligned}
$$

where $U=(c, s)$ solves the approximate system (19). The following lemma shows some estimates that will guarantee the continuity and compactness of $\mathcal{F}$ below.

Lemma 2.4. Let $c_{0}, b_{1,0}, b_{2,0}, b_{3,0}, b_{4,0} \in L^{\infty}\left(\Omega_{c y t}\right)$ and $s_{0}, b_{5,0} \in L^{\infty}\left(\Omega_{s r}\right)$, then for any $(\hat{c}, \hat{s}) \in L^{2}\left(\Omega_{c y t, T}\right) \times$ $L^{2}\left(\Omega_{s r, T}\right)$, the system (19) has a unique solution. Moreover, there exists a constant $C>0$ depending on the initial conditions, such that

$$
\begin{aligned}
& \|c\|_{L^{\infty}\left(0, T ; L^{2}\left(\Omega_{c y t}\right)\right)}+\|c\|_{L^{2}\left(0, T ; H^{1}\left(\Omega_{c y t}\right)\right)} \leq C, \\
& \left\|b_{i}\right\|_{L^{\infty}\left(0, T ; L^{2}\left(\Omega_{c y t}\right)\right)}+\left\|b_{i}\right\|_{L^{2}\left(0, T ; H^{1}\left(\Omega_{c y t}\right)\right)} \leq C \text { for } i=1, \ldots, 4, \\
& \|s\|_{L^{\infty}\left(0, T ; L^{2}\left(\Omega_{s r}\right)\right)}+\|s\|_{L^{2}\left(0, T ; H^{1}\left(\Omega_{s r}\right)\right)} \leq C \\
& \left\|b_{5}\right\|_{L^{\infty}\left(0, T ; L^{2}\left(\Omega_{s r}\right)\right)}+\left\|b_{5}\right\|_{L^{2}\left(0, T ; H^{1}\left(\Omega_{s r}\right)\right)} \leq C .
\end{aligned}
$$

Proof. For the proof it suffices to use assumptions on the initial conditions and the definition of functions $R_{i}$ (for $i=1, \ldots, 5$ ) combined with the general result for parabolic equations (see for e.g. for more details [15]).

To prove the existence and uniqueness of (16), we apply now the Schauder fixed point theorem to the map $\mathcal{F}$. The goal is to prove that such map has a fixed-point. First, we notice that $\mathcal{F}$ is well defined. To satisfy the hypothesis of the fixed point theorem, we show that $\mathcal{F}$ is continuous and compact mapping.

Firstly, we prove the continuity of $\mathcal{F}$. Let $\left(\hat{c}_{n}, \hat{s}_{n}\right)$ be a sequence in $\mathcal{A}$ and $(\hat{c}, \hat{s}) \in \mathcal{A}$ be such that

$$
\left(\hat{c}_{n}, \hat{s}_{n}\right) \rightarrow(\hat{c}, \hat{s}) \quad \text { in } L^{2}\left(\Omega_{c y t, T}\right) \times L^{2}\left(\Omega_{s r, T}\right),
$$

as $n \rightarrow \infty$. Let us then define

$$
\left(c_{n}, s_{n}\right)=\mathcal{F}\left(\hat{c}_{n}, \hat{s}_{n}\right)
$$


i.e., $\left(c_{n}, s_{n}\right)$ is the solution of (19) associated with the sequence $\left(\hat{c}_{n}, \hat{s}_{n}\right)$. The objective is to show that:

$$
\left(c_{n}, s_{n}\right) \text { converges to } \mathcal{F}(\hat{c}, \hat{s}),
$$

in $L^{2}\left(\Omega_{c y t, T}\right) \times L^{2}\left(\Omega_{s r, T}\right)$. We start with the following lemma:

Lemma 2.5. Let $\left(c_{n}, b_{1, n}, b_{2, n}, b_{3, n}, b_{4, n}, s_{n}, b_{5, n}\right)_{n}$ be the solution to problem (19). Then

(i) There exists a constant $C>0$ such that for $i=1, \ldots, 4$

$$
0 \leq c_{n}\left(x_{c}, t\right), b_{i, n}\left(x_{c}, t\right), s_{n}\left(x_{s}, t\right), b_{5, n}\left(x_{s}, t\right) \leq C,
$$

for a.e. $\left(x_{c}, t\right) \in \Omega_{c y t, T}$ and $\left(x_{s}, t\right) \in \Omega_{s r, T}$.

(ii) The sequence $\left(c_{n}, b_{1, n}, b_{2, n}, b_{3, n}, b_{4, n}, b_{5, n}, s_{n}\right)_{n}$ is bounded in $\left(L^{2}\left(0, T ; H^{1}\left(\Omega_{c y t}\right)\right) \cap L^{\infty}\left(0, T ; L^{2}\left(\Omega_{c y t}\right)\right)\right)^{5} \times\left(L^{2}\left(0, T ; H^{1}\left(\Omega_{s r}\right)\right) \cap L^{\infty}\left(0, T ; L^{2}\left(\Omega_{s r}\right)\right)\right)^{2}$.

(iii) The sequence $\left(c_{n}, b_{1, n}, b_{2, n}, b_{3, n},, b_{4, n}, b_{5, n} s_{n}\right)_{n}$ is relatively compact in $\left(L^{2}\left(\Omega_{c y t, T}\right)\right)^{5} \times\left(L^{2}\left(\Omega_{s r, T}\right)\right)^{2}$.

Proof. To prove (i), we multiply the first and the third equations in (19) by $-c_{n}^{-}$and $-s_{n}^{-}$(where $\alpha^{-}:=$ $(\alpha-|\alpha|) / 2)$ and we integrate over $\Omega_{c y t}$ and $\Omega_{s r}$, respectively. The result is

$$
\begin{array}{r}
\frac{1}{2} \frac{d}{d t} \int_{\Omega_{c y t}}\left|c_{n}^{-}\right|^{2} d x-\int_{\Omega_{c y t}} D_{c}\left(\hat{c}_{n}\right) \nabla c_{n} \cdot \nabla c_{n}^{-} d x+\int_{\Gamma_{r y r}} g\left(s_{n}-c_{n}\right) c_{n}^{-} d \sigma d t \\
=\sum_{i=1}^{4} \int_{\Omega_{c y t}} R_{i, \varepsilon}\left(c_{n}^{+}, b_{i, n}^{+}\right) c_{n}^{-} d x \leq 0,
\end{array}
$$

and

$$
\begin{array}{r}
\frac{1}{2} \frac{d}{d t} \int_{\Omega_{s r}}\left|s_{n}^{-}\right|^{2} d x-\int_{\Omega_{s r}} D_{s}\left(\hat{s}_{n}\right) \nabla s_{n} \cdot \nabla s_{n}^{-} d x-\int_{\Gamma_{r y r}} g\left(s_{n}-c_{n}\right) s_{n}^{-} d \sigma d t \\
=\int_{\Omega_{s r}} R_{s, \varepsilon}\left(c_{n}^{+}, b_{i, n}^{+}\right) s_{n}^{-} d x \leq 0 .
\end{array}
$$

Using this and (17), we deduce

$$
\frac{1}{2} \frac{d}{d t}\left\|c_{n}^{-}\right\|_{L^{2}\left(\Omega_{c y t}\right)}^{2}+\frac{1}{2} \frac{d}{d t}\left\|s_{n}^{-}\right\|_{L^{2}\left(\Omega_{s r}\right)}^{2}+\iint_{\Gamma_{r y r}} g(s-c)\left(c_{n}^{-}-s_{n}^{-}\right) d \sigma d t \leq 0 .
$$

Observe that the function $(\cdot)^{-}$is a decreasing function. This implies

$$
\iint_{\Gamma_{r y r, T}} g\left(s_{n}-c_{n}\right)\left(c_{n}^{-}-s_{n}^{-}\right) d \sigma d t \geq 0 .
$$

Therefore we obtain

$$
\frac{1}{2} \frac{d}{d t}\left\|c_{n}^{-}\right\|_{L^{2}\left(\Omega_{c y t}\right)}^{2}+\frac{1}{2} \frac{d}{d t}\left\|s_{n}^{-}\right\|_{L^{2}\left(\Omega_{s r}\right)}^{2} \leq 0
$$

Since the initial condition $\left(c_{0}, s_{0}\right)$ is nonnegative we conclude

$$
c_{n}\left(t, x_{c}\right), s_{n}\left(t, x_{s}\right) \geq 0 \text { for a.e. }\left(t, x_{c}\right) \in \Omega_{c y t, T} \text { and }\left(t, x_{s}\right) \in \Omega_{s r, T} .
$$

Reasoning similarly we get the nonnegativity of $b_{i, n}$ for $i=1, \ldots, 5$.

In the next step we prove first (recall that $B_{i}$ is the total concentration of the buffer)

$$
b_{i, n}\left(t, x_{c}\right) \leq B_{i} \text { and } b_{5, n}\left(t, x_{s}\right) \leq B_{5} \text { for a.e. }\left(t, x_{c}\right) \in \Omega_{c y t, T} \text { and }\left(t, x_{s}\right) \in \Omega_{s r, T},
$$


for $i=1, \ldots, 4$. For this, we multiply $\left(b_{i, n}-B_{i}\right)^{+}\left(\right.$where $\left.\alpha^{+}:=(\alpha+|\alpha|) / 2\right)$ by the corresponding equation of $b_{i, n}$ in (19) and we integrate over $\Omega_{c y t}$ for $i=1, \ldots, 4$ and $\Omega_{s r}$ for $i=5$, respectively, to deduce from the definition of $R_{i, \varepsilon}$

$$
\begin{gathered}
\frac{1}{2} \sum_{i=1}^{4} \frac{d}{d t} \|\left(( b _ { i , n } - B _ { i } ) ^ { + } \| _ { L ^ { 2 } ( \Omega _ { c y t } ) } ^ { 2 } + \frac { 1 } { 2 } \frac { d } { d t } \| \left(\left(b_{5 ; n}-B_{5}\right)^{+}\left\|_{L^{2}\left(\Omega_{s r}\right)}^{2}+\sum_{i=1}^{3}\right\| \nabla\left(b_{i, n}-B_{i}\right)^{+} \|_{L^{2}\left(\Omega_{s r, T}\right)}^{2}\right.\right. \\
\quad=\sum_{i=1}^{4} \iint_{\Omega_{c y t, T}} R_{i, \varepsilon}\left(\hat{c}_{n}, b_{i, n}\right)\left(b_{i, n}-B_{i}\right)^{+} d x d t+\iint_{\Omega_{s r}} R_{5, \varepsilon}\left(\hat{s}_{n}, b_{5, n}\right)\left(b_{5, n}-B_{5}\right)^{+} d x d t \leq 0 .
\end{gathered}
$$

Using this and the Gronwall lemma we get (23). To complete the prove, let us define a super solution to the equations of calcium :

$$
\left\{\begin{array}{l}
\frac{d}{d t} M(t)=-\sum_{i=1}^{5} R_{i}(M(t), 0)=-\sum_{i=1}^{5} k_{i}^{o n} M(t) B_{i} \\
M(0)=\left\|c_{0}\right\|_{L^{\infty}\left(\Omega_{c y t}\right)}+\left\|s_{0}\right\|_{L^{\infty}\left(\Omega_{s r}\right)}
\end{array}\right.
$$

Now, we multiply the first and the third equations in (19) by $\left(c_{n}-M(t)\right)^{+}$and $\left(s_{n}-M(t)\right)^{+}$, respectively, and we integrate over $\Omega_{c y t}$ and $\Omega_{s r}$, respectively, and we sum the resulting equations to obtain

$$
\begin{aligned}
\frac{1}{2} \frac{d}{d t} \| & \left(c_{n}-M(t)\right)^{+}\left\|_{L^{2}\left(\Omega_{c y t}\right)}^{2}+\frac{1}{2} \frac{d}{d t}\right\|\left(s_{n}-M(t)\right)^{+}\left\|_{L^{2}\left(\Omega_{s r}\right)}^{2}+\right\| \nabla\left(c_{n}-M(t)\right)^{+} \|_{L^{2}\left(\Omega_{c y t, T}\right)}^{2} \\
& +\left\|\nabla\left(s_{n}-M(t)\right)^{+}\right\|_{L^{2}\left(\Omega_{s r, T}\right)}^{2}+\iint_{\Gamma_{r y r}} g\left(s_{n}-c_{n}\right)\left(\left(s_{n}-M(t)\right)^{+}-\left(c_{n}-M(t)\right)^{+}\right) d \sigma d t \\
& =\sum_{i=1}^{4} \iint_{\Omega_{c y t, T}}\left(R_{i, \varepsilon}(M(t), 0)-R_{i, \varepsilon}\left(c_{n}, b_{i, n}\right)\right)\left(c_{n}-M(t)\right)^{+} d x d t \\
& +\iint_{\Omega_{s r}}\left(R_{s, \varepsilon}(M(t), 0)-R_{s, \varepsilon}\left(s_{n}, b_{5, n}\right)\right)\left(s_{n}-M(t)\right)^{+} d x d t \\
& =\sum_{i=1}^{4} \iint_{\Omega_{c y t, T}}\left(R_{i, \varepsilon}(M(t), 0)-R_{i, \varepsilon}\left(c_{n}, 0\right)+R_{i, \varepsilon}\left(c_{n}, 0\right)-R_{i, \varepsilon}\left(c_{n}, b_{i, n}\right)\right)\left(c_{n}-M(t)\right)^{+} d x d t \\
& +\iint_{\Omega_{s r}}\left(R_{s, \varepsilon}(M(t), 0)-R_{s, \varepsilon}\left(s_{n}, 0\right)+R_{s, \varepsilon}\left(s_{n}, 0\right)-R_{s, \varepsilon}\left(s_{n}, b_{5, n}\right)\right)\left(s_{n}-M(t)\right)^{+} d x d t
\end{aligned}
$$

By monotonicity of $(\cdot-M(t))^{+}$we have $\iint_{\Gamma_{r y r}, T} g\left(s_{n}-c_{n}\right)\left(\left(s_{n}-M(t)\right)^{+}-\left(c_{n}-M(t)\right)^{+}\right) d \sigma d t \geq 0$. For the right hand terms, we assume that $c_{n} \geq M(t)$ and $s_{n} \geq M(t)$. Then, by monotonicity of $R_{i, \varepsilon}$ we conclude that :

$$
\frac{1}{2} \frac{d}{d t}\left\|\left(c_{n}-M(t)\right)^{+}\right\|_{L^{2}\left(\Omega_{c y t}\right)}^{2}+\frac{1}{2} \frac{d}{d t}\left\|\left(s_{n}-M(t)\right)^{+}\right\|_{L^{2}\left(\Omega_{s r}\right)}^{2} \leq 0
$$

Therefore, we have $c_{n}\left(t, x_{c}\right), s_{n}\left(t, x_{s}\right) \leq M(t)$ for all $\left(t, x_{c}\right) \in \Omega_{c y t, T}$ and $\left(t, x_{s}\right) \in \Omega_{s r, T}$. Moreover, an application of the Gronwall lemma on (24) we obtain

$$
M(t) \leq M(0) e^{-t C} \leq\left\|c_{0}\right\|_{L^{\infty}\left(\Omega_{c y t}\right)}+\left\|s_{0}\right\|_{L^{\infty}\left(\Omega_{s r}\right)} \text { with } C=\sum_{i=1}^{5} B_{i} k_{i}^{o n} .
$$

To prove (ii), we multiply the first, the second, the third, the fourth and the fifth equation in (19) by $c_{n}$, $b_{i, n}$ (for $\left.i=1, \ldots, 4\right), s_{n}$ and $b_{5, n}$ respectively. Herein, we replace the solution $\left(c, b_{1}, b_{2}, b_{3}, b_{4}, s, b_{5}\right)$ by $\left(c_{n}, b_{1, n}, b_{2, n}, b_{3, n}, b_{4, n}, s_{n}, b_{5, n}\right)$. Next, we sum and integrate the resulting equation on $(0, T)$, on $\Omega_{c y t}$ for $\left(c_{n}, b_{1, n}, b_{2, n}, b_{3, n}, b_{4, n}\right)$ and on $\Omega_{s r}$ for $\left(s_{n}, b_{5, n}\right)$. Hence the Gronwall lemma and Poincare inequality yields

$$
\left\|\left(c_{n}, b_{1, n}, b_{2, n}, b_{3, n}, b_{4, n}\right)\right\|_{\left(L^{2}\left(0, T ; H^{1}\left(\Omega_{c y t}\right)\right) \cap L^{\infty}\left(0, T ; L^{2}\left(\Omega_{c y t}\right)\right)\right)^{5}} \leq C,
$$

and

$$
\left\|\left(s_{n}, b_{5, n}\right)\right\|_{\left(L^{2}\left(0, T ; H^{1}\left(\Omega_{s r}\right)\right) \cap L^{\infty}\left(0, T ; L^{2}\left(\Omega_{s r}\right)\right)\right)^{2}} \leq C,
$$


for some constant $C>0$ not depending on $n$. Finally, note that from (i) and (ii) we get easily for $i=1, \ldots, 4$

$$
\begin{aligned}
& \left\|\partial_{t} c_{n}\right\|_{L^{2}\left(0, T ;\left(H^{1}\left(\Omega_{c y t}\right)\right)^{\prime}\right)}+\left\|\partial_{t} s_{n}\right\|_{L^{2}\left(0, T ;\left(H^{1}\left(\Omega_{s r}\right)\right)^{\prime}\right)} \\
& \quad+\left\|\partial_{t} b_{i, n}\right\|_{L^{2}\left(0, T ;\left(H^{1}\left(\Omega_{c y t}\right)\right)^{\prime}\right)}+\left\|\partial_{t} b_{5, n}\right\|_{L^{2}\left(0, T ;\left(H^{1}\left(\Omega_{s r}\right)\right)^{\prime}\right)} \leq C,
\end{aligned}
$$

for some constant $C>0$ not depending on $n$. Therefore, (iii) is a consequence of this, (ii) and the compactness argument (see for e.g. [30] for more details). This concludes the proof of Lemma 2.5.

In summary, Lemma 2.5 and the theory of compact sets [30], imply that there exists $\left(c, b_{1}, b_{2}, b_{3}, b_{4}, s, b_{5}\right) \in$ $\left(L^{2}\left(0, T ; H^{1}\left(\Omega_{c y t}\right)\right)\right)^{5} \times\left(L^{2}\left(0, T ; H^{1}\left(\Omega_{s r}\right)\right)\right)^{2}$ such that, up to extracting subsequences if necessary,

$$
\left\{\begin{array}{l}
\left(c_{n}, b_{i, n}, b_{2, n}, b_{3, n},, b_{4, n}, b_{5, n}, s_{n}\right) \rightarrow\left(c, b_{1}, b_{2}, b_{3}, b_{4}, b_{5}, s\right) \text { in }\left(L^{2}\left(\Omega_{c y t, T}\right)\right)^{5} \times\left(L^{2}\left(\Omega_{s r, T}\right)\right)^{2} \\
\text { strongly and a.e., and in }\left(L^{2}\left(0, T ; H^{1}\left(\Omega_{c y t}\right)\right)\right)^{5} \times\left(L^{2}\left(0, T ; H^{1}\left(\Omega_{s r}\right)\right)\right)^{2} \text { weakly. }
\end{array}\right.
$$

Consequently, the continuity of $\mathcal{F}$ on $\mathcal{A}$ holds.

Moreover, using again Lemma 2.5 and the theory of compact sets [30], we get the compactness of the map $\mathcal{F}(\mathcal{A}) \hookrightarrow L^{2}\left(\Omega_{c y t, T}\right) \times L^{2}\left(\Omega_{s r, T}\right)$. Then, thanks to Schauder fixed-point theorem, the operator $\mathcal{F}$ has a fixed point $\left(c_{\varepsilon}, s_{\varepsilon}\right)$. That is, there exists a solution to the approximate problem (19). That is there exists a solution to

$$
\begin{aligned}
& \int_{0}^{T}\left\langle\partial_{t} c_{\varepsilon}, \varphi_{c}\right\rangle d t+\iint_{\Omega_{c y t, T}} D_{c}\left(c_{\varepsilon}\right) \nabla c_{\varepsilon} \cdot \nabla \varphi_{c} d x d t-\int_{0}^{T} \int_{\Gamma_{r y r}} g\left(s_{\varepsilon}-c_{\varepsilon}\right) \varphi_{c} \sigma d t \\
& =-\iint_{\Omega_{c y t, T}} \sum_{i=1}^{4} R_{i, \varepsilon}\left(c_{\varepsilon}, b_{i, \varepsilon}\right) \varphi_{c} d x d t \\
& \int_{0}^{T}\left\langle\partial_{t} s_{\varepsilon}, \varphi_{s}\right\rangle d t+\iint_{\Omega_{s r, T}} D_{s}\left(s_{\varepsilon}\right) \nabla s_{\varepsilon} \cdot \nabla \varphi_{s} d x d t+\int_{0}^{T} \int_{\Gamma_{r y r}} g\left(s_{\varepsilon}-c_{\varepsilon}\right) \varphi_{s} \sigma d t \\
& =-\iint_{\Omega_{s r, T}} R_{s, \varepsilon}\left(s_{\varepsilon}, b_{5, \varepsilon}\right) \varphi_{s} d x d t, \\
& \int_{0}^{T}\left\langle\partial_{t} b_{i, \varepsilon}, \varphi_{i}\right\rangle d t+D_{b_{i}} \iint_{\Omega_{c y t, T}} \nabla b_{i, \varepsilon} \cdot \nabla \varphi_{i} d x d t=\iint_{\Omega_{c y t, T}} R_{i, \varepsilon}\left(c_{\varepsilon}, b_{i, \varepsilon}\right) \varphi_{i} d x d t \\
& \int_{0}^{T}\left\langle\partial_{t} b_{5, \varepsilon}, \varphi_{5}\right\rangle d t+D_{b_{5}} \iint_{\Omega_{s r}, T} \nabla b_{5, \varepsilon} \cdot \nabla \varphi_{5} d x d t=\iint_{\Omega_{s r, T}} R_{5, \varepsilon}\left(c_{\varepsilon}, b_{5, \varepsilon}\right) \varphi_{5} d x d t
\end{aligned}
$$

for all $\varphi_{c}, \varphi_{i} \in L^{2}\left(0, T ; H^{1}\left(\Omega_{c y t}\right)\right)$ and $\varphi_{s}, \varphi_{5} \in L^{2}\left(0, T ; H^{1}\left(\Omega_{s r}\right)\right)$ for $i=1, \ldots, 4$.

\subsection{Existence of weak solutions}

We have shown in Section 2.1 that problem (27) admits a solution $\left(c_{\varepsilon}, b_{1, \varepsilon}, b_{2, \varepsilon}, b_{3, \varepsilon}, b_{4, \varepsilon}, s_{\varepsilon}, b_{5, \varepsilon}\right)$. The goal in this subsection is to send the regularization parameter $\varepsilon$ to zero in sequences of such solutions to obtain weak solutions of the original system (16). Observe that, for each fixed $\varepsilon>0$, we have shown the existence of a solution. $\left(c_{\varepsilon}, b_{i, \varepsilon}, b_{2, \varepsilon}, b_{3, \varepsilon}, b_{4, \varepsilon}, s_{\varepsilon}, b_{5, \varepsilon}\right)$ to $(27)$ such that for $i=1, \ldots, 4$

$$
0 \leq c_{\varepsilon}\left(x_{c}, t\right), b_{i, \varepsilon}\left(x_{c}, t\right), s_{\varepsilon}\left(x_{s}, t\right), b_{5, \varepsilon}\left(x_{s}, t\right) \leq M
$$

for a.e. $\left(x_{c}, t\right) \in \Omega_{c y t, T}$ and $\left(x_{s}, t\right) \in \Omega_{s r, T}$. Herein, the constant $M>0$ is not depending on $\varepsilon$. Now, we substitute $\varphi_{c}=c_{\varepsilon}, \varphi_{s}=s_{\varepsilon}, \varphi_{i}=b_{i, \varepsilon}$ in $(27)$ for $i=1, \ldots, 5$, and we work exactly as in the proof of ( $\left.i i\right)$ in 
Lemma 2.5 , to obtain for $i=1, \ldots, 4$

$$
\begin{array}{rl}
\sup _{0 \leq t \leq T} \int_{\Omega_{c y t}}\left|c_{\varepsilon}(x, t)\right|^{2} & d x+\sup _{0 \leq t \leq T} \int_{\Omega_{s r}}\left|s_{\varepsilon}(x, t)\right|^{2} d x \\
& +\sup _{0 \leq t \leq T} \int_{\Omega_{c y t}}\left|b_{i, \varepsilon}(x, t)\right|^{2} d x+\sup _{0 \leq t \leq T} \int_{\Omega_{s r}}\left|b_{5, \varepsilon}(x, t)\right|^{2} d x \leq C,
\end{array}
$$

and

$$
\begin{aligned}
\iint_{\Omega_{c y t, T}}\left|\nabla c_{\varepsilon}(x, t)\right|^{2} d x d t+\iint_{\Omega_{s r, T}}\left|\nabla s_{\varepsilon}(x, t)\right|^{2} d x d t \\
+\iint_{\Omega_{c y t, T}}\left|\nabla b_{i, \varepsilon}(x, t)\right|^{2} d x d t+\iint_{\Omega_{s r, T}}\left|\nabla b_{5, \varepsilon}(x, t)\right|^{2} d x d t \leq C,
\end{aligned}
$$

for some constant $C>0$ independent of $\varepsilon$. Repeating the steps of the proof of $($ iii $)$ in Lemma 2.5, we derive the bound for $i=1, \ldots, 4$,

$$
\begin{aligned}
& \left\|\partial_{t} c_{\varepsilon}\right\|_{L^{2}\left(0, T ;\left(H^{1}\left(\Omega_{c y t}\right)\right)^{\prime}\right)}+\left\|\partial_{t} s_{\varepsilon}\right\|_{L^{2}\left(0, T ;\left(H^{1}\left(\Omega_{s r}\right)\right)^{\prime}\right)} \\
& \quad+\left\|\partial_{t} b_{i, \varepsilon}\right\|_{L^{2}\left(0, T ;\left(H^{1}\left(\Omega_{c y t}\right)\right)^{\prime}\right)}+\left\|\partial_{t} b_{5, \varepsilon}\right\|_{L^{2}\left(0, T ;\left(H^{1}\left(\Omega_{s r}\right)\right)^{\prime}\right)} \leq C,
\end{aligned}
$$

for some constant $C>0$. Then, combining (28), (29) and (30) with standard compactness results (cf. [30]) we can extract subsequences, which we do not relabel, such that, as $\varepsilon$ goes to 0 ,

$$
\left\{\begin{array}{l}
\left(c_{\varepsilon}, b_{i, \varepsilon}, s_{\varepsilon}, b_{5, \varepsilon}\right) \rightarrow\left(c, b_{i}, s, b_{5}\right) \text { in }\left(L^{2}\left(\Omega_{c y t, T}\right)^{2}\right) \times\left(L^{2}\left(\Omega_{s r, T}\right)\right)^{2} \\
\text { strongly and a.e., and in }\left(L^{2}\left(0, T ; H^{1}\left(\Omega_{c y t}\right)\right)\right)^{2} \times\left(L^{2}\left(0, T ; H^{1}\left(\Omega_{s r}\right)\right)\right)^{2} \text { weakly } \\
\left(\partial_{t} c_{\varepsilon}, \partial_{t} b_{i, \varepsilon}\right) \rightarrow\left(\partial_{t} c, \partial_{t} b_{i}\right) \text { weakly in }\left(L^{2}\left(0, T ;\left(H^{1}\left(\Omega_{c y t}\right)\right)^{\prime}\right)\right)^{2} \\
\left(\partial_{t} s_{\varepsilon}, \partial_{t} b_{5, \varepsilon}\right) \rightarrow\left(\partial_{t} s, \partial_{t} b_{5}\right) \text { weakly in }\left(L^{2}\left(0, T ;\left(H^{1}\left(\Omega_{s r}\right)\right)^{\prime}\right)\right)^{2}
\end{array}\right.
$$

for $i=1 \ldots, 4$. This result, together with the weak- $\star$ convergences in $L^{\infty}$ of $\left(c_{\varepsilon}, b_{1, \varepsilon}, b_{2, \varepsilon}, b_{3, \varepsilon}, s_{\varepsilon}\right)$ to $\left(c, b_{1}, b_{2}, b_{3}\right.$, $s)$, yields

$$
\left\{\begin{array}{l}
\left(c_{\varepsilon}, b_{1, \varepsilon}, b_{2, \varepsilon}, b_{3, \varepsilon}, b_{4, \varepsilon}\right) \rightarrow\left(c, b_{1}, b_{2}, b_{3}, b_{4}\right),\left(s_{\varepsilon}, b_{5, \varepsilon}\right) \rightarrow\left(s, b_{5}\right) \text { strongly in }\left(L^{p}\left(\Omega_{c y t, T}\right)\right)^{5}, \\
\text { and in }\left(L^{p}\left(\Omega_{s r, T}\right)\right)^{2}, \text { respectively, for } 1 \leq p<\infty,
\end{array}\right.
$$

which in turn implies that for $i=1, \ldots, 5$

$$
R_{i, \varepsilon}\left(c_{\varepsilon}, b_{i, \varepsilon}\right) \rightarrow R_{i}\left(c, b_{i}\right), \quad R_{s, \varepsilon}\left(s_{\varepsilon}, b_{5, \varepsilon}\right) \rightarrow R_{s}\left(s, b_{5}\right)
$$

a.e. and strongly in $L^{p}$ for $1 \leq p<\infty$. As a consequence of (31), (32) and (33), it is clear that as $\varepsilon \rightarrow 0$ in (27), we can identify the limit as the weak solution from Definition 2.2 .

Remark 2. Note that, since the interaction terms are Lipschitz (recall that our solutions are uniformly bounded in $L^{\infty}$ ) and the positivity of diffusion function, the uniqueness of weak solution can be obtained easily (for a similar proof see [1]).

\section{Numerical approximation}

In this section we construct our finite element scheme for the system (16). We establish several a priori (discrete energy) estimates for our scheme and compactness of discrete solutions which eventually will imply the desired convergence results to the unique weak solution.

\subsection{The semi-discrete scheme}

We consider a quasi-uniform family $\mathcal{T}_{i, h}$ of triangulations of $\bar{\Omega}_{i}$ by tetrahedra $K$, for $i=c y t, s r$. The parameter $h$ has the sense of an upper bound for the maximum diameter of the control volumes in $\mathcal{T}_{i, h}$ for $i=c y t, s r$. For 
a given an integer $k \geq 0$ and $S \subset \mathbb{R}^{3}$, we denote by $\mathcal{P}_{k}(S)$ the space of polynomial functions defined in $S$ of total degree up to $k$. Next, we define the finite element subspaces $\mathcal{V}_{i, h}$ by

$$
\mathcal{V}_{i, h}=\left\{m_{h} \in H^{1}\left(\Omega_{i}\right):\left.m_{h}\right|_{K} \in \mathcal{P}_{1}(K) \forall K \in \mathcal{T}_{i, h}\right\}
$$

where for any $K \in \mathcal{T}_{i, h}\left(\Omega_{i}\right)$ for $i=c y t, s r$. respectively.

We consider the appropriate $L^{2}$-projections of the initial condition $\left(c_{h}^{0}, b_{i, h}^{0}\right)=\mathbb{P}_{\mathcal{V}_{c y t, h}}\left(c_{0}, b_{i, 0}\right)($ for $i=1, \ldots, 4)$ and $\left(s_{h}^{0}, b_{5, h}^{0}\right)=\mathbb{P}_{\mathcal{V}_{h}}\left(s_{0}, b_{5,0}\right)$. To advance the numerical solution from $t_{n-1}$ to $t_{n}=t_{n-1}+\delta t(\delta t=T / N)$, we use the following implicit finite element scheme:

For $n \in\{1, \ldots, N\}$, find $\left(c_{h}^{n}, b_{1, h}^{n}, b_{2, h}^{n}, b_{3, h}^{n}, b_{4, h}^{n}\right) \in\left(\mathcal{V}_{c y t, h}\right)^{5}$ and $\left(s_{h}^{n}, b_{5, h}^{n}\right) \in\left(\mathcal{V}_{s r, h}\right)^{2}$ such that

$$
\begin{aligned}
& \left(\frac{c_{h}^{n}-c_{h}^{n-1}}{\delta t}, \varphi_{c}\right)_{\Omega_{c y t}}+\int_{\Omega_{c y t}} D_{c}\left(c_{h}^{n}\right) \nabla c_{h}^{n} \cdot \nabla \varphi_{c} d x-\int_{\Gamma_{r y r}} g\left(s_{h}^{n}-c_{h}^{n}\right) \varphi_{c} d \sigma \\
& =-\sum_{i=1}^{4}\left(R_{i}\left(c_{h}^{n}, b_{i, h}^{n}\right), \varphi_{c}\right)_{\Omega_{c y t}}, \\
& \left(\frac{s_{h}^{n}-s_{h}^{n-1}}{\delta t}, \varphi_{s}\right)_{\Omega_{s r}}+\int_{\Omega_{s r}} D_{s}\left(s_{h}^{n}\right) \nabla s_{h}^{n} \cdot \nabla \varphi_{s} d x+\int_{\Gamma_{r y r}} g\left(s_{h}^{n}-c_{h}^{n}\right) \varphi_{s} d \sigma \\
& =-\left(R_{5}\left(s_{h}^{n}, b_{5, h}^{n}\right), \varphi_{s}\right)_{\Omega_{s r}}, \\
& \left(\frac{b_{i, h}^{n}-b_{i, h}^{n-1}}{\delta t}, \varphi_{i}\right)_{\Omega_{c y t}}+\int_{\Omega_{c y t}} D_{b_{i}} \nabla b_{i, h}^{n} \cdot \nabla \varphi_{i} d x=\left(R_{i}\left(c_{h}^{n}, b_{i, h}^{n}\right), \varphi_{i}\right)_{\Omega_{c y t}}, \\
& \left(\frac{b_{5, h}^{n}-b_{5, h}^{n-1}}{\delta t}, \varphi_{5}+\int_{\Omega_{s r}} D_{b_{5}} \nabla b_{5, h}^{n} \cdot \nabla \varphi_{5} d x=\left(R_{5}\left(s_{h}^{n}, b_{5, h}^{n}\right), \varphi_{5}\right)_{\Omega_{s r}},\right.
\end{aligned}
$$

for all $\varphi_{c}, \varphi_{i} \in \mathcal{V}_{c y t, h}$ and $\varphi_{s}, \varphi_{5} \in \mathcal{V}_{s r, h}$ for $i=1, \ldots, 4$

\subsection{Numerical convergence analysis}

Note that the problem of proving uniform boundedness of the discrete finite element solutions is still an open problem (recall that the positive and negative cuts test functions are not admissible in finite element context). So, comparing to the continuous case, we assume that the initial conditions $\left(c_{0}, b_{1,0}, b_{2,0}, b_{3,0},, b_{4,0}\right)$ and $\left(s_{0}, b_{5,0}\right)$ are only bounded in $L^{2}$.

The convergence of solutions generated by the scheme (34) is next established following two main lemmas. The first one states $L^{\infty}\left(L^{2}\right) \cap L^{2}\left(H^{1}\right)$ boundedness of the discrete concentrations and buffers $\left(c_{h}, b_{1, h}, b_{2, h}, b_{3, h}, b_{4, h}\right.$, $\left.s_{h}, b_{5, h}\right)$. We establish several a priori (discrete energy) estimates, which eventually will imply the desired convergence results.

Remark 3. In the proof of Lemmas 3.1 and 3.2 below, we will use the following Gagliardo-Nierenberg inequality: For $\Omega \subset \mathbb{R}^{3}$ there exists a constant $G$ such that for $u \in H^{1}(\Omega)$

$$
\|u\|_{L^{q}(\Omega)} \leq G\|u\|_{H^{1}(\Omega)}^{\alpha}\|u\|_{L^{2}(\Omega)}^{1-\alpha} \text { for } \frac{1}{q}=\frac{3-2 \alpha}{6} \text { and } \alpha \in(0,1) .
$$


Lemma 3.1. Let $\left(c_{h}^{n}, b_{1, h}^{n}, b_{2, h}^{n}, b_{3, h}^{n}, b_{4, h}^{n}, s_{h}^{n}, b_{5, h}^{n}\right)$ be part of the solution of (34). Then there exists a constant $C>0$ depending on $\left\|c_{0}\right\|_{L^{2}\left(\Omega_{c y t}\right)},\left\|b_{i, 0}\right\|_{L^{2}\left(\Omega_{c y t}\right)}$, and $\left\|s_{0}\right\|_{L^{2}\left(\Omega_{s r}\right)},\left\|b_{5,0}\right\|_{L^{2}\left(\Omega_{s r}\right)}$ such that for $i=1, \ldots, 4$

$$
\begin{gathered}
\left\|\left(c_{h}, b_{i, h}\right)\right\|_{L^{\infty}\left(0, T ; L^{2}\left(\Omega_{c y t}, \mathbb{R}^{2}\right)\right)}+\left\|\left(s_{h}, b_{5, h}\right)\right\|_{L^{\infty}\left(0, T ; L^{2}\left(\Omega_{c y t}, \mathbb{R}^{2}\right)\right)} \leq C, \\
\left\|\nabla c_{h}\right\|_{L^{2}\left(\Omega_{T, c y t}\right)}+\sum_{i=1}^{3}\left\|\nabla b_{i, h}\right\|_{L^{2}\left(\Omega_{T, c y t}\right)}+\left\|\nabla s_{h}\right\|_{L^{2}\left(\Omega_{T, s r}\right)} \leq C
\end{gathered}
$$

Proof. We use (34) with $\phi_{c}=c_{h}^{n}, \phi_{s}=-s_{h}^{n}$ and $\phi_{i}=b_{i, h}^{n}$ (for $\left.i=1, \ldots, 5\right)$, and we sum over $n=1, \ldots, k$ for all $1<k \leq N$. The result is

$$
\begin{aligned}
& \frac{1}{2}\left(\left(c_{h}^{k}, c_{h}^{k}\right)_{\Omega_{c y t}}+\left(s_{h}^{k}, s_{h}^{k}\right)_{\Omega_{s r}}+\sum_{i=1}^{4}\left(b_{i, h}^{k}, b_{i, h}^{k}\right)_{\Omega_{c y t}}+\left(b_{5, h}^{k}, b_{5, h}^{k}\right)_{\Omega_{s r}}\right) \\
& +D^{\min }\left(\sum_{n=1}^{k} \int_{\Omega_{c y t}}\left|\nabla c_{h}^{n}\right|^{2}+\sum_{n=1}^{k} \int_{\Omega_{s r}}\left|\nabla s_{h}^{n}\right|^{2}\right) \\
& +\int_{\Gamma_{r y r}} g\left|s_{h}^{n}-c_{h}^{n}\right|^{2} d \sigma \\
& \leq \frac{1}{2}\left(\left(c_{0, h}, c_{0, h}\right)_{\Omega_{c y t}}+\left(s_{0, h}, s_{0, h}\right)_{\Omega_{s r}}+\sum_{i=1}^{4}\left(b_{i, 0, h}, b_{i, 0, h}\right)_{\Omega_{c y t}}+\left(b_{5,0, h}, b_{5,0, h}\right)_{\Omega_{s r}}\right) \\
& +C_{1}\left(\sum_{n=1}^{k} \int_{\Omega_{c y t}}\left(\left|c_{h}^{n}\right|^{2}+\sum_{i=1}^{4}\left|b_{i, h}^{n}\right|^{2}\right)+\sum_{n=1}^{k} \int_{\Omega_{s r}}\left(\left|s_{h}^{n}\right|^{2}+\left|b_{5, h}^{n}\right|^{2}\right)\right) \\
& +C_{2}\left(\sum_{i=1}^{4} \sum_{n=1}^{k} \int_{\Omega_{c y t}}\left(\left|\left(c_{h}^{n}\right)^{2} b_{i, h}^{n}\right|+\left|c_{h}^{n}\left(b_{i, h}^{n}\right)^{2}\right|\right)+\sum_{n=1}^{k} \int_{\Omega_{s r}}\left(\left|\left(s_{h}^{n}\right)^{2} b_{5, h}^{n}\right|+\left|s_{h}^{n}\left(b_{5, h}^{n}\right)^{2}\right|\right)\right) \\
& :=\frac{1}{2} I_{1}+C_{1} I_{2}+C_{3} I_{3},
\end{aligned}
$$

for some constant $C_{1}, C_{2}>0$. Herein, we have used the positivity of $D_{c}$ and $D_{s}$, and the convexity inequality

$$
a^{n}\left(a^{n}-a^{n-1}\right) \geq \frac{\left|a^{n}\right|^{2}}{2}-\frac{\left|a^{n-1}\right|^{2}}{2} .
$$

Observe that since the initial conditions are bounded in $L^{2}$, we get

$$
I_{1} \leq C_{3}
$$

for some constant $C_{3}>0$. Regarding the term $I_{3}$, we use Gagliardo-Nieremberg inequality (35) for $q=4$, Poincaré inequality and Young inequality to deduce for $i=1, \ldots, 4$

$$
\begin{aligned}
\sum_{n=1}^{k} \int_{\Omega_{c y t}}\left|\left(c_{h}^{n}\right)^{2} b_{i, h}^{n}\right| & \leq \sum_{n=1}^{k}\left(\left\|c_{h}^{n}\right\|_{L^{2}\left(\Omega_{c y t}\right)}\left\|c_{h}^{n}\right\|_{L^{4}\left(\Omega_{c y t}\right)}\left\|b_{i, h}^{n}\right\|_{L^{4}\left(\Omega_{c y t}\right)}\right) \\
& \leq G \sum_{n=1}^{k}\left(\left\|c_{h}^{n}\right\|_{L^{2}\left(\Omega_{c y t}\right)}^{5 / 4}\left\|c_{h}^{n}\right\|_{H^{1}\left(\Omega_{c y t}\right)}^{3 / 4}\left\|b_{i, h}^{n}\right\|_{L^{2}\left(\Omega_{c y t}\right)}^{1 / 4}\left\|b_{i, h}^{n}\right\|_{H^{1}\left(\Omega_{c y t}\right)}^{3 / 4}\right) \\
& \leq \frac{D^{\min }}{2} \sum_{n=1}^{k}\left\|c_{h}^{n}\right\|_{H^{1}\left(\Omega_{c y t}\right)}\left\|b_{i, h}^{n}\right\|_{H^{1}\left(\Omega_{c y t}\right)}+C_{4} \sum_{n=1}^{k}\left\|c_{h}^{n}\right\|_{L^{2}\left(\Omega_{c y t}\right)}^{5}\left\|b_{i, h}^{n}\right\|_{L^{2}\left(\Omega_{c y t}\right)} \\
& \leq \frac{D^{\min }}{4} \sum_{n=1}^{k}\left(\left\|c_{h}^{n}\right\|_{H^{1}\left(\Omega_{c y t}\right)}^{2}+\left\|b_{i, h}^{n}\right\|_{H^{1}\left(\Omega_{c y t}\right)}^{2}\right) \\
& +C_{5} \sum_{n=1}^{k}\left(\left\|c_{h}^{n}\right\|_{L^{2}\left(\Omega_{c y t}\right)}^{6}+\left\|b_{i, h}^{n}\right\|_{L^{2}\left(\Omega_{c y t}\right)}^{6}\right),
\end{aligned}
$$


for some constants $C_{4}, C_{5}>0$. Similarly, we get for for $i=1, \ldots, 4$

$$
\begin{aligned}
& \sum_{n=1}^{k} \int_{\Omega_{c y t}}\left|c_{h}^{n}\left(b_{i, h}^{n}\right)^{2}\right| \leq \frac{D^{\mathrm{min}}}{4} \sum_{n=1}^{k}\left(\left\|c_{h}^{n}\right\|_{H^{1}\left(\Omega_{c y t}\right)}^{2}+\left\|b_{i, h}^{n}\right\|_{H^{1}\left(\Omega_{c y t}\right)}^{2}\right) \\
&+C_{6} \sum_{n=1}^{k}\left(\left\|c_{h}^{n}\right\|_{L^{2}\left(\Omega_{c y t}\right)}^{6}+\left\|b_{i, h}^{n}\right\|_{L^{2}\left(\Omega_{c y t}\right)}^{6}\right), \\
& \sum_{n=1}^{k} \int_{\Omega_{s r}}\left|\left(s_{h}^{n}\right)^{2} b_{5, h}^{n}\right| \leq \frac{D^{\mathrm{min}}}{4} \sum_{n=1}^{k}\left(\left\|s_{h}^{n}\right\|_{H^{1}\left(\Omega_{s r}\right)}^{2}+\left\|b_{5, h}^{n}\right\|_{H^{1}\left(\Omega_{s r}\right)}^{2}\right) \\
&+C_{7} \sum_{n=1}^{k}\left(\left\|s_{h}^{n}\right\|_{L^{2}\left(\Omega_{s r}\right)}^{6}+\left\|b_{5, h}^{n}\right\|_{L^{2}\left(\Omega_{s r}\right)}^{6}\right),
\end{aligned}
$$

and

$$
\begin{aligned}
\sum_{n=1}^{k} \int_{\Omega_{c y t}}\left|s_{h}^{n}\left(b_{5, h}^{n}\right)^{2}\right| \leq \frac{D^{\min }}{4} \sum_{n=1}^{k}( & \left.\left\|s_{h}^{n}\right\|_{H^{1}\left(\Omega_{s r}\right)}^{2}+\left\|b_{5, h}^{n}\right\|_{H^{1}\left(\Omega_{s r}\right)}^{2}\right) \\
& +C_{8} \sum_{n=1}^{k}\left(\left\|s_{h}^{n}\right\|_{L^{2}\left(\Omega_{s r}\right)}^{6}+\left\|b_{5, h}^{n}\right\|_{L^{2}\left(\Omega_{s r}\right)}^{6}\right),
\end{aligned}
$$

for some constants $C_{6}, C_{7}, C_{8}>0$. Collecting the previous inequalities, we obtain

$$
\begin{gathered}
\left(\int_{\Omega_{c y t}}\left(\left|c_{h}^{k}\right|^{2}+\sum_{i=1}^{4}\left|b_{i, h}^{k}\right|^{2}\right)+\int_{\Omega_{s r}}\left(\left|s_{h}^{k}\right|^{2}+\left|b_{5, h}^{k}\right|^{2}\right)\right) \\
+\frac{D^{\min }}{2}\left(\sum_{n=1}^{k} \int_{\Omega_{c y t}}\left|\nabla c_{h}^{n}\right|^{2}+\sum_{n=1}^{k} \int_{\Omega_{s r}}\left|\nabla s_{h}^{n}\right|^{2}\right) \\
\leq 2 C_{3}+2 C_{1} \sum_{n=1}^{k}\left(\int_{\Omega_{c y t}}\left(\left|c_{h}^{n}\right|^{2}+\sum_{i=1}^{4}\left|b_{i, h}^{n}\right|^{2}\right)+\int_{\Omega_{s r}}\left(\left|s_{h}^{n}\right|^{2}+\left|b_{5, h}^{n}\right|^{2}\right)\right) \\
+C_{9}\left(\sum_{n=1}^{k}\left(\left\|c_{h}^{n}\right\|_{L^{2}\left(\Omega_{c y t}\right)}^{6}+\sum_{i=1}^{4}\left\|b_{i, h}^{n}\right\|_{L^{2}\left(\Omega_{c y t}\right)}^{6}\right)\right. \\
\left.\quad+\sum_{n=1}^{k}\left(\left\|s_{h}^{n}\right\|_{L^{2}\left(\Omega_{s r}\right)}^{6}+\sum_{i=1}^{4}\left\|b_{5, h}^{n}\right\|_{L^{2}\left(\Omega_{s r}\right)}^{6}\right)\right) \\
\leq 2 C_{3}+C_{10} \sum_{n=1}^{k}\left[\int_{\Omega_{c y t}}\left(\left|c_{h}^{n}\right|^{2}+\sum_{i=1}^{4}\left|b_{i, h}^{n}\right|^{2}\right)+\int_{\Omega_{s r}}\left(\left|s_{h}^{n}\right|^{2}+\left|b_{5, h}^{n}\right|^{2}\right)\right] \\
\times\left[1+\int_{\Omega_{c y t}}\left(\left|c_{h}^{n}\right|^{2}+\sum_{i=1}^{4}\left|b_{i, h}^{n}\right|^{2}\right)+\int_{\Omega_{s r}}\left(\left|s_{h}^{n}\right|^{2}+\left|b_{5, h}^{n}\right|^{2}\right)\right]^{2},
\end{gathered}
$$

for some constants $C_{9}, C_{10}>0$. Now we set

$$
U:=1+\int_{\Omega_{c y t}}\left(\left|c_{h}\right|^{2}+\sum_{i=1}^{4}\left|b_{i, h}\right|^{2}\right)+\int_{\Omega_{s r}}\left(\left|s_{h}\right|^{2}+\left|b_{5, h}\right|^{2}\right) .
$$


Observe that (37) implies

$$
U^{k} \leq C_{11}+C_{10} \sum_{n=1}^{k}\left(U^{n}\right)^{3} \text { for every } 1<k \leq N,
$$

and for some constant $C_{11}>0$. Therefore by the discrete Gronwall inequality [32] to $U$, yields

$$
\begin{aligned}
\int_{\Omega_{c y t}}\left(\left|c_{h}\right|^{2}+\sum_{i=1}^{4}\left|b_{i, h}\right|^{2}\right) & +\int_{\Omega_{s r}}\left(\left|s_{h}\right|^{2}+\left|b_{5, h}\right|^{2}\right) \\
\leq U(t) & \leq \operatorname{Const}\left(\left\|\left(c_{0}, b_{i, 0}, s_{0}, b_{5,0}\right)\right\|\right)_{L^{2}\left(\Omega_{c y t}, \mathbb{R}^{2}\right) \times L^{2}\left(\Omega_{s r}, \mathbb{R}^{2}\right)},
\end{aligned}
$$

for $i=1, \ldots, 4$. Therefore, we get the first estimate of (36). Using this and (37) we get the second part of estimate (36). This concludes the proof of Lemma 3.1.

Now, we consider $\left(\bar{c}_{h}, \bar{b}_{i, h}\right)$ and $\left(\bar{s}_{h}, \bar{b}_{5, h}\right)$ the piecewise affine in $t$ functions in $\left(W^{1, \infty}\left([0 ; T] ; V_{c y t, h}\right)\right)^{2}$ and in $\left(W^{1, \infty}\left([0 ; T] ; V_{s r, h}\right)\right)^{2}$, respectively, and interpolating $\left(c_{h}^{n}, b_{i, h}^{n}\right)_{n=0, \ldots, N} \subset\left(V_{c y t, h}\right)^{2}$ and $\left(s_{h}^{n}, b_{5, h}^{n}\right)_{n=0, \ldots, N} \subset$ $\left(V_{s r, h}\right)^{2}$ at the points $(n \Delta t)_{n=0, \ldots, N}$ (recall the interpolation scheme $\bar{n}_{h}=n_{h}^{n-1}+\frac{t-t_{n-1}}{\Delta t}\left(n_{h}^{n}-n_{h}^{n-1}\right)$ for $\left.n=c, s, b_{i}\right)$ for $i=1, \ldots, 4$. Then we have

$$
\begin{cases}\partial_{t} \bar{c}_{h}=\operatorname{div}\left(D_{c}\left(c_{h}\right) \nabla c_{h}\right)-\sum_{i=1}^{4} R_{i}\left(c_{h}, b_{i, h}\right), & \text { in } \Omega_{c y t, T} \\ \partial_{t} \bar{b}_{i, h}=\operatorname{div}\left(D_{b_{i}} \nabla b_{i, h}\right)+R_{i}\left(c_{h}, b_{i, h}\right), i \in\{1, \ldots, 4\}, & \text { in } \Omega_{c y t, T} \\ \partial_{t} \bar{s}_{h}=\operatorname{div}\left(D_{s}\left(s_{h}\right) \nabla s_{h}\right)-R_{5}\left(s_{h}, b_{5, h}\right), & \text { in } \Omega_{s r, T} \\ \partial_{t} \bar{b}_{5, h}=\operatorname{div}\left(D_{b_{5}} \nabla b_{5, h}\right)+R_{5}\left(s_{h}, b_{5, h}\right), & \text { in } \Omega_{s r, T}\end{cases}
$$

In the next lemma, we establish the relative compactness in $L^{2}$ of the sequences $\left(c_{h}, b_{1, h}, b_{2, h}, b_{3, h}, b_{4, h}, s_{h}, b_{5, h}\right)$, which is achieved by constructing space and time translates and using the a priori estimates given above.

Lemma 3.2. There exist a constant $C>0$ depending on initial conditions, $\Omega_{s r}, \Omega_{c y t}$ and $T$ such that for $i=1, \ldots, 4$

$$
\left\|\left(\bar{c}_{h}, \bar{b}_{i, h}\right)\right\|_{\left(L^{2}\left(0, T ; H^{1}\left(\Omega_{c y t}\right)\right)\right)^{2}}+\left\|\left(\bar{s}_{h}, \bar{b}_{5, h}\right)\right\|_{\left(L^{2}\left(0, T ; H^{1}\left(\Omega_{s r}\right)\right)\right)^{2}} \leq C,
$$

and

$$
\begin{gathered}
\left|\int_{0}^{T}\left\langle\partial_{t} \bar{c}_{h}, \varphi_{c}\right\rangle_{c y t} d t\right|+\sum_{i=1}^{4}\left|\int_{0}^{T}\left\langle\partial_{t} \bar{b}_{i, h}, \varphi_{i}\right\rangle_{c y t} d t\right| \\
+\left|\int_{0}^{T}\left\langle\partial_{t} \bar{s}_{h}, \varphi_{s}\right\rangle_{s r} d t\right|+\left|\int_{0}^{T}\left\langle\partial_{t} \bar{b}_{5, h}, \varphi_{5}\right\rangle_{s r} d t\right| \\
\leq C\left(\left\|\varphi_{c}\right\|_{L^{2}\left(0, T ; H^{1}\left(\Omega_{c y t}\right)\right)}+\sum_{i=1}^{4}\left\|\varphi_{i}\right\|_{L^{2}\left(0, T ; H^{1}\left(\Omega_{c y t}\right)\right)}\right. \\
\left.+\left\|\varphi_{s}\right\|_{L^{2}\left(0, T ; H^{1}\left(\Omega_{s r}\right)\right)}+\left\|\varphi_{5}\right\|_{L^{2}\left(0, T ; H^{1}\left(\Omega_{s r}\right)\right)}\right),
\end{gathered}
$$

for any $\varphi_{c}, \varphi_{i} \in L^{2}\left(0, T ; H^{1}\left(\Omega_{c y t}\right)\right)$ and $\varphi_{s}, \varphi_{5} \in L^{2}\left(0, T ; H^{1}\left(\Omega_{s r}\right)\right)$. 
Proof. First, we begin by proving (39) but this is the consequence of the definition of $\left(\bar{c}_{h}, \bar{s}_{h}, \bar{b}_{i, h}\right)($ for $i=$ $1, \ldots, 5)$ and Lemma 3.1 .

The next part of the prove is devoted to (40). Let $\varphi_{c} \in L^{2}\left(0, T ; H^{1}(\Omega)\right)$. Using (38), Lemma 3.1 GagliardoNieremberg inequality, Poincaré inequality and Young inequality, we obtain

$$
\begin{aligned}
& \left|\int_{0}^{T}\left\langle\partial_{t} \bar{c}_{h}, \varphi_{c}\right\rangle_{c y t} d t\right| \\
& =\left|\int_{0}^{T}\left(D_{c}\left(c_{h}\right) \nabla c_{h}, \varphi_{c}\right)_{c y t} d t-\sum_{i=1}^{4} \int_{0}^{T}\left(R_{i}\left(c_{h}, b_{i, h}\right), \varphi_{c}\right)_{c y t} d t\right| \\
& \leq D^{\max }\left\|\nabla c_{h}\right\|_{L^{2}\left(\Omega_{c y t, T}\right)}\left\|\nabla \varphi_{c}\right\|_{L^{2}\left(\Omega_{c y t, T}\right)}+C_{12}\left(\left\|c_{h}\right\|_{L^{2}\left(\Omega_{c y t, T}\right)}\right. \\
& \left.\quad+\sum_{i=1}^{4}\left\|b_{i, h}\right\|_{L^{2}\left(\Omega_{c y t, T}\right)}\right)\left\|\varphi_{c}\right\|_{L^{2}\left(\Omega_{c y t, T}\right)}+C_{13} \sum_{i=1}^{4} \int_{0}^{T}\left(\left|c_{h}, b_{i, h}\right|,\left|\varphi_{c}\right|\right)_{c y t} d t \\
& \leq C_{14}\left\|\varphi_{c}\right\|_{L^{2}\left(0, T ; H^{1}\left(\Omega_{c y t}\right)\right)}+C_{13} \sum_{i=1}^{4} I_{i},
\end{aligned}
$$

for some constants $C_{12}, C_{13}, C_{14}>0$. Regarding the integral $I_{i}$, we use Gagliardo-Nieremberg inequality (35) (for $q=3$ ) and Young inequality to obtain for $i=1, \ldots, 4$

$$
\begin{aligned}
& I_{i} \leq \int_{0}^{T}\left\|c_{h}\right\|_{L^{2}\left(\Omega_{c y t}\right)}\left\|b_{i, h}\right\|_{L^{3}\left(\Omega_{c y t}\right)}\left\|\varphi_{c}\right\|_{L^{6}\left(\Omega_{c y t}\right)} d t \\
& \leq G\left\|c_{h}\right\|_{L^{\infty}\left(0, T ; L^{2}\left(\Omega_{c y t}\right)\right)} \sqrt{\left\|b_{i, h}\right\|_{L^{\infty}\left(0, T ; L^{2}\left(\Omega_{c y t}\right)\right)}} \int_{0}^{T} \sqrt{\left\|b_{i, h}\right\|_{H^{1}\left(\Omega_{c y t}\right)}}\left\|\varphi_{c}\right\|_{L^{6}\left(\Omega_{c y t}\right)} d t \\
& \leq G\left\|c_{h}\right\|_{L^{\infty}\left(0, T ; L^{2}\left(\Omega_{c y t}\right)\right)} \sqrt{\left\|b_{i, h}\right\|_{L^{\infty}\left(0, T ; L^{2}\left(\Omega_{c y t}\right)\right)}} \\
& \times \sqrt{\left\|b_{i, h}\right\|_{L^{1}\left(0, T ; H^{1}\left(\Omega_{c y t}\right)\right.}}\left\|\varphi_{c}\right\|_{L^{2}\left(0, T ; L^{6}\left(\Omega_{c y t}\right)\right)} d t .
\end{aligned}
$$

Now we use Lemma 3.1 and the continuous embedding of $L^{2}\left(0, T ; H^{1}\left(\Omega_{c y t}\right)\right)$ into $L^{2}\left(0, T ; L^{6}\left(\Omega_{\text {cyt }}\right)\right)$ to get from $(42)$

$$
I_{i} \leq C\left\|\varphi_{c}\right\|_{L^{2}\left(0, T ; H^{1}\left(\Omega_{c y t}\right)\right)},
$$

for some constant $C>0$, for $i=1, \ldots, 4$. From this and (41) we deduce the bound

$$
\left\|\partial_{t} \bar{c}_{h}\right\|_{L^{2}\left(0, T ;\left(H^{1}(\Omega)\right)^{\prime}\right)} \leq C
$$

Reasoning similarly, we get $(40)$ for $\left(\bar{b}_{i, h}, \bar{s}_{h}\right)$ for $i=1, \ldots, 5$, therefore finishing our proof.

Observe that from the definition of $\left(\bar{c}_{h}, \bar{s}_{h}, \bar{b}_{i, h}\right)$ together with (34) eventually implies that

$$
\begin{aligned}
& \| \bar{c}_{h}- c_{h}\left\|_{L^{2}\left(\Omega_{c y t, T}\right)}^{2}+\sum_{i=1}^{4}\right\| \bar{b}_{i, h}-b_{i, h} \|_{L^{2}\left(\Omega_{c y t, T}\right)}^{2} \\
& \quad \leq \sum_{n=1}^{N} \Delta t\left\|c_{h}^{n}-c_{h}^{n-1}\right\|_{L^{2}\left(\Omega_{c y t}\right)}^{2}+\sum_{i=1}^{4}\left\|b_{i, h}^{n}-b_{i, h}^{n-1}\right\|_{L^{2}\left(\Omega_{c y t}\right)}^{2} \leq \mathcal{C}(\Delta t) \quad \rightarrow 0 \text { as } \Delta t \rightarrow 0,
\end{aligned}
$$


and

$$
\begin{aligned}
& \| \bar{s}_{h}- s_{h}\left\|_{L^{2}\left(\Omega_{s r, T}\right)}^{2}+\right\| \bar{b}_{5, h}-\bar{b}_{5, h} \|_{L^{2}\left(\Omega_{s r, T}\right)}^{2} \\
& \quad \leq \sum_{n=1}^{N} \Delta t\left\|s_{h}^{n}-s_{h}^{n-1}\right\|_{L^{2}\left(\Omega_{s r}\right)}^{2}+\left\|b_{5, h}^{n}-b_{5, h}^{n-1}\right\|_{L^{2}\left(\Omega_{s r}\right)}^{2} \leq \mathcal{C}(\Delta t) \quad \rightarrow 0 \text { as } \Delta t \rightarrow 0 .
\end{aligned}
$$

Therefore, from Lemma 3.2 and standard compactness results (see for e.g. [30]), there exists a subsequence of $\left(c_{h}, s_{h}, b_{1, h}, b_{2, h}, b_{3, h}, b_{4, h}, b_{5, h}\right)$ not relabeled, such that, as $h \rightarrow 0$,

$$
\left\{\begin{array}{l}
\left(c_{h}, b_{1, h}, b_{2, h}, b_{3, h}, b_{4, h}\right) \rightarrow\left(c, b_{1}, b_{2}, b_{3}, b_{4}\right) \text { strongly and a.e. in }\left(L^{2}\left(\Omega_{c y t, T}\right)^{5}\right. \\
\left(s, b_{5, h}\right) \rightarrow\left(s, b_{5}\right) \text { strongly and a.e. in } L^{2}\left(\Omega_{s r, T}\right)^{2} \\
\left(c_{h}, b_{1, h}, b_{2, h}, b_{3, h}, b_{4, h}\right) \rightarrow\left(c, b_{1}, b_{2}, b_{3}, b_{4}\right) \text { weakly in } L^{2}\left(0, T ; H^{1}\left(\Omega_{c y t}\right)\right)^{5} \\
\left(s, b_{5, h}\right) \rightarrow\left(s, b_{5}\right) \text { weakly in } L^{2}\left(0, T ; H^{1}\left(\Omega_{s r}\right)\right)^{2}
\end{array}\right.
$$

Finally, from the convergence in (44) we can identify the limit $\left(c, b_{1}, b_{2}, b_{3}, b_{4}, s, b_{5}\right)$ as the weak solution of (16), and the convergence result is summarized as follows.

Theorem 3.3. Assume that condition (17) holds. If $c_{0}, b_{1,0}, b_{2,0}, b_{3,0}, b_{4,0} \in L^{2}\left(\Omega_{c y t}\right)$ and $s_{0}, b_{5,0} \in L^{2}\left(\Omega_{s r}\right)$, then the finite element solution $\left(c_{h}, b_{1, h}, b_{2, h}, b_{3, h}, b_{4, h}, s_{h}, b_{5, h}\right)$, generated by (34), converges along a subsequence to $\left(c, b_{1}, b_{2}, b_{3}, b_{4}, s, b_{5}\right)$ as $h, \delta t \rightarrow 0$, where $\left(c, b_{1}, b_{2}, b_{3}, b_{4}, s, b_{5}\right)$ is a weak solution of (16) in the sense of Definition 2.1.

\section{Numerical SIMULATION}

In this section, we present some numerical simulations in several experiments with different parameters. In the first experiment, we investigate the diffusion function to catch the accurate calcium wave front characteristics using imaging observations as reference [7]. In the next experiment, we analyze the influence of the RyR conductance on calcium front wave propagation in cytosolic domain, followed by an investigation on pathological consequences on the cellular scale. Finally, we extend our simulation results in three dimensional framework and we discuss the phenomenological behavior of calcium spark through RyR.

To approximate the solution, we consider the finite element framework defined in Section 3 along with Euler implicit time discretization with time step $\delta t=10^{-5} \mathrm{~s}$. The mesh parameters are determined using mesh adaptation to deal with high gradient of the solutions that came from the non uniform distribution of $b_{4}$ and $b_{5}$ (See Figure 3b below). For calcium initial concentration, we take $c_{0}=0.14$ in $\Omega_{c y t}$ and $s_{0}=1.3 \times 10^{3}$ in $\Omega_{s r}$. For buffers initial concentration in $\Omega_{c y t}$ we put $b_{1,0}=318 \times 10^{-3}, b_{2,0}=471 \times 10^{-3}, b_{3,0}=2.82, b_{4,0}=13.2$ and the initial concentration of CSQN in $\Omega_{s r}$ is $b_{5,0}=24.6 \times 10^{3}$. The values of previous parameters are provided in $\mu M$. We present the diffusion coefficient and interaction parameters (see [11] and the references therein) in Table 1. Almost all parameters have been discovered in cardiac cell medium.

\begin{tabular}{|l|l|l|l|l|l|}
\hline Diffusion coefficient & $\overline{D_{c}}$ & $\overline{D_{s}}$ & $D_{b_{1}}$ & $D_{b_{2}}$ & $D_{b_{3}}$ \\
\hline Value & 220 & 73.3 & 140 & 25 & 42 \\
\hline \hline Total concentration & $B_{1}$ & $B_{2}$ & $B_{3}$ & $B_{4}$ & $B_{5}$ \\
\hline Value & 140 & 25 & 42 & 42 & 42 \\
\hline \hline Off rates & $k_{1}^{\text {off }}$ & $k_{2}^{\text {off }}$ & $k_{3}^{\text {off }}$ & $k_{4}^{\text {off }}$ & $k_{5}^{\text {off }}$ \\
\hline Value & 45 & 0.238 & 0.110 & 0.0196 & 0.065 \\
\hline \hline On rates & $k_{1}^{\text {on }}$ & $k_{2}^{\text {on }}$ & $k_{3}^{\text {on }}$ & $k_{4}^{\text {on }}$ & $k_{5}^{\text {on }}$ \\
\hline Value & 255 & 34 & 110 & 32.7 & 102 \\
\hline
\end{tabular}

TABLE 1. Total concentration, reaction and diffusion rates in a cardiac cell. 
Remark 4. Table 1 shows experimental data related to cardiac cell. The units are presented as follows : The diffusion coefficient $\left(D_{l}\right)$ unit is $10^{3} \mathrm{~nm}^{2} \mathrm{~ms}^{-1}$ and the total mass unit $\left(B_{i}\right)$ is $\mu M$. The $k_{i}^{\text {on }}$ unit is $10^{-3} \mathrm{~ms}^{-1} \mu \mathrm{m}^{-1}$ and $k_{i}^{\text {off }}$ unit is $m s^{-1}$. The diffusion of buffers $b_{4}$ and $b_{5}$ are taken very small.

\section{D numerical simulation of calcium model}

In cell medium, we mention that fixed buffers are not uniformly distributed (see [11] for more details) which would induce a higher gradient of the solution in some regions. Hence, we consider a random distribution of buffers and we adapt the mesh generation (see Figure 3) to achieve a finer approximation in the critical regions. The mesh adaptation is performed using a maximal edge length $h_{\max }=0.3$ on both computational domain with maximal number of vertexes $120 \times 10^{3}$ (resp. $200 \times 10^{3}$ ) for cytosolic domain (resp. sarcoplasmic domain).

Figure 4 shows the two dimensional $\mathrm{Ca}^{2+}$-distribution for a time instant of $t=1 \mathrm{~ms}$ after the calcium spark. The concentration dependent diffusion coefficient is given by :

$$
D_{s}(v)=0.1(1+v) \text { and } D_{c}(u)=\overline{D_{c}}\left(0.5+u^{m}\right)
$$

with different values of $m$ (A similar formulation is given in [6]).

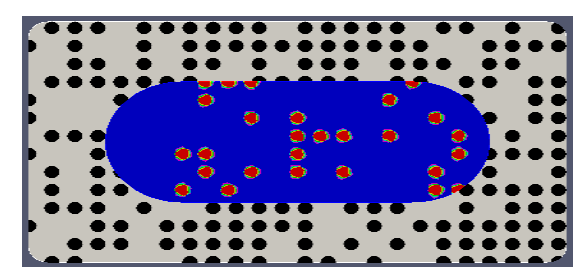

(a)

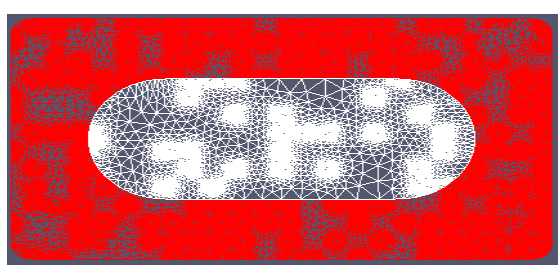

(b)

FIGURE 3. The left hand-side figure represents the distribution of $b_{4}$ and $b_{5}$. The right hand-side figure shows the Meshing used during the simulation
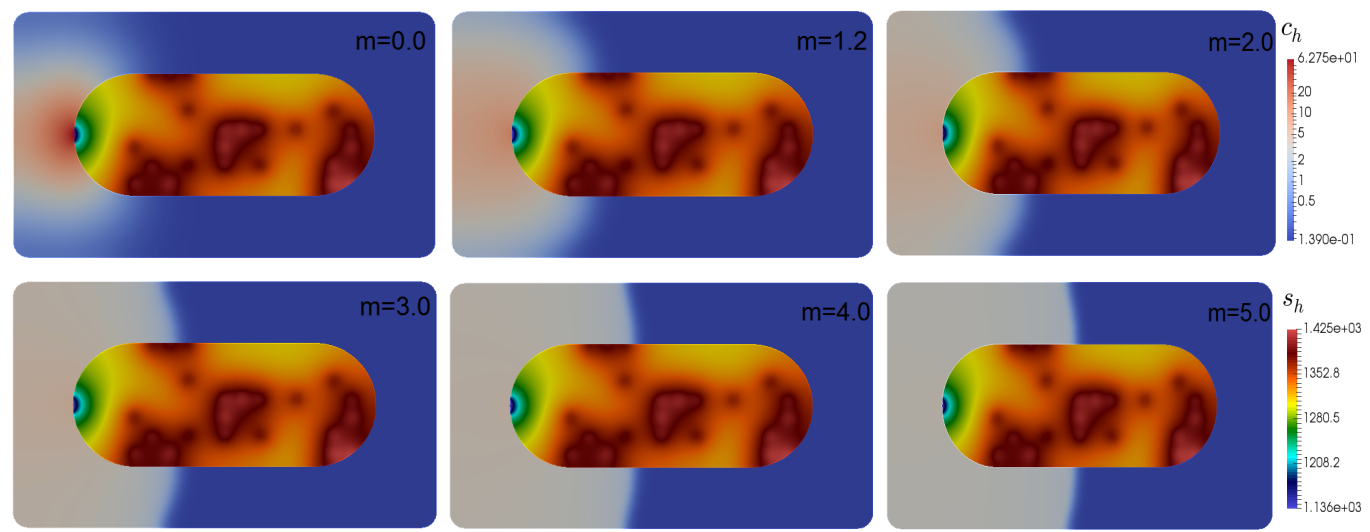

FIGURE 4. The Graphical representation of the approximated solution in Cytosolic domain with non constant diffusion coefficient with $m=0, \ldots, 5$ at $t=50 \times 10^{-5} \mathrm{~s}$. 


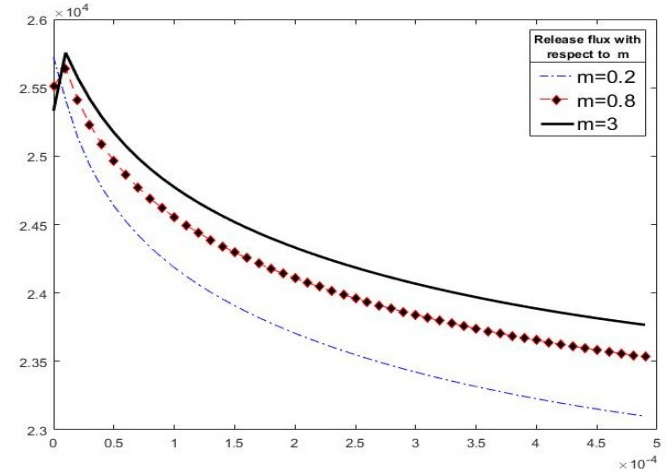

(a) Release flux with $g=100$

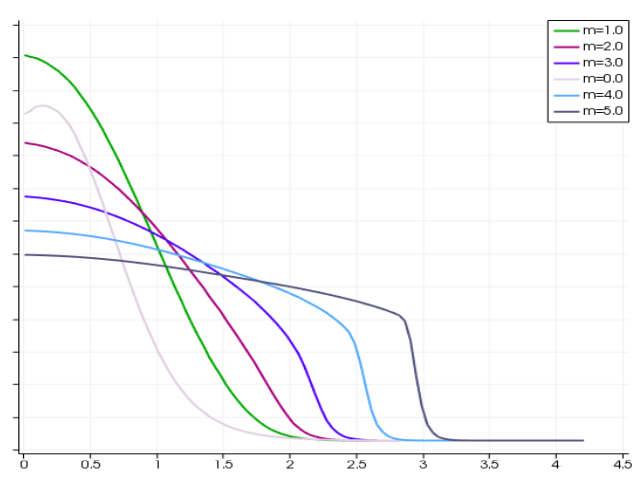

(b) Spatial calcium distibution through a line

Figure 5. Figure 5a shows temporal dynamics of release flux (i.e. $\int_{\Gamma_{r y r}} g(s-c)$ ) and Figure $5 \mathrm{~b}$ represents a one dimensional calcium spatial distribution for various values of $m=1, \ldots, 5$

By changing $m$ values from 0 to 5 (see Figure 5b), we observe that $C a^{2+}$ wavefront displays an ellipsoidal shape with a fast decaying near to the front wave. In imaging results from [7], we observe the same phenomenon due to the heavy fixed buffering in the cell medium that decreases the calcium diffusion in the low concentration areas. We mention also the explosive increasing of release flux at the beginning of calcium spark which has been confirmed experimentally in [34]. Hence, The non constant diffusion coefficient induces the same phenomenon. The larger $m$ is taken more the explosive increasing is important.
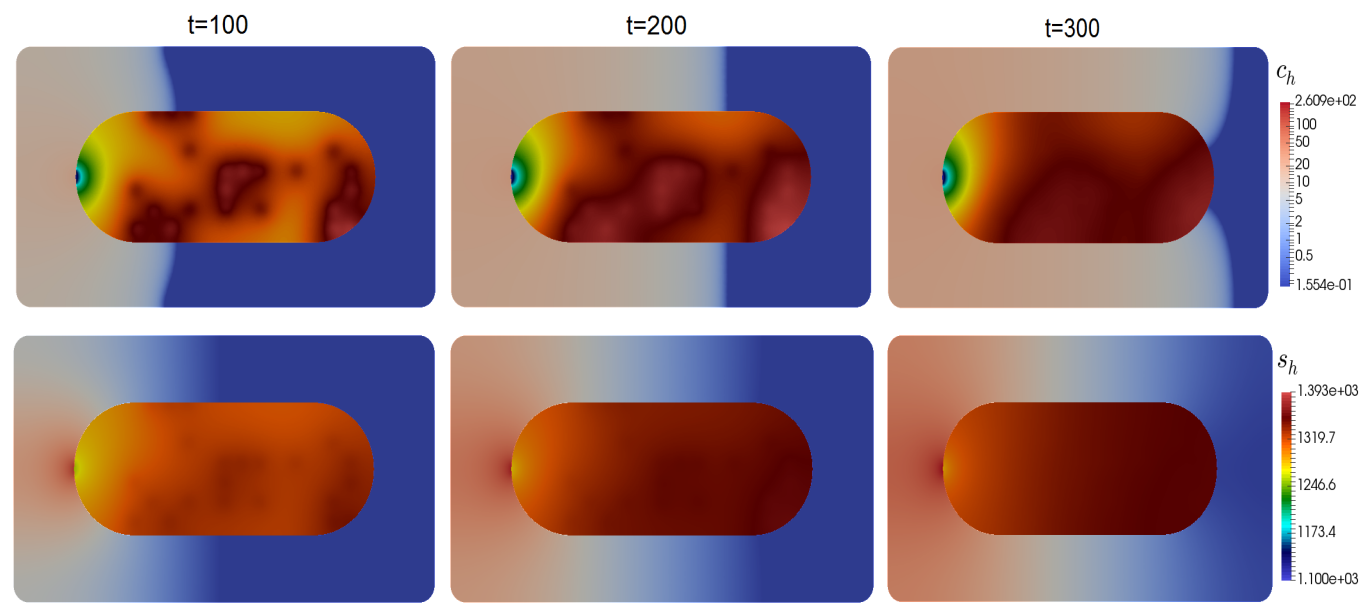

FIGURE 6. The figures on the down side represent the evolution of calcium distribution in the linear case. The figures on the up side devoted to the nonlinear case with $m=3$

To understand the wavefront differences between linear $\left(D_{c}(u)=1.5 \bar{D}_{c}\right.$ and $\left.D_{s}(v)=\bar{D}_{s}\right)$ and nonlinear case $(m=3)$, Figure 6 shows the evolution $\mathrm{Ca}^{2+}$-distribution in both cases. In the second row, we observe a large propagating front wave moving away from the $\mathrm{Ca}^{2+}$ highest concentration around RyR (similar observation shown in [11]) because of the linear diffusion with the simple Fickian law. On the upside, the propagating front wave is much smaller and clear in this case. Moreover, this affects the release flux through RyR, see Figure 5a. 


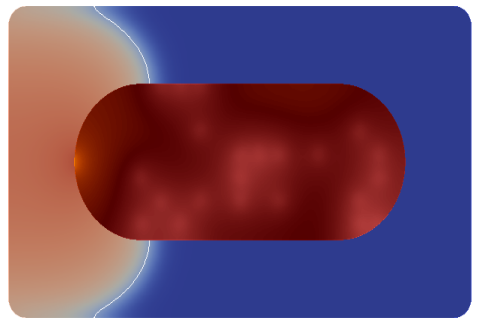

(a)

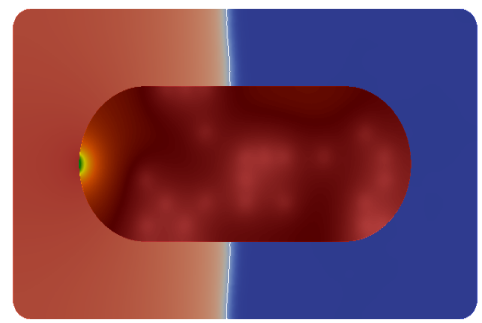

(b)

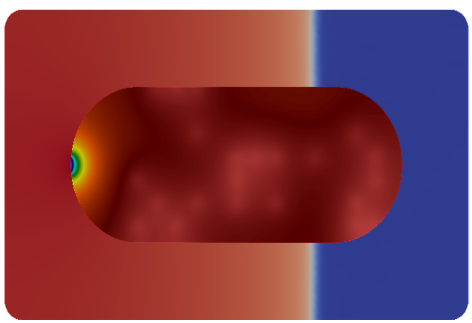

(c)

FIGURE 7. The influence of RyR conductance on calcium wave front velocity. The values of RyR conductance $g$ in Figures 7a, 7b and 7c are respectively 50,150 and 300

Figure 7, shows the influence of RyR conductance on calcium wave front velocity. Figure 7c shows a faster wave front than in Figure 7a case. Pathological behaviors of RyR influences directly the calcium wave velocity and consequently influences the heart beat rate. In other cases some perturbations on heavy buffering protein (CSQN) inside the SR influence the release flux through RyR. In this case, the universal RyR conductance should be corrected to regularize the calcium wave by regularizing the release flux through ionic channels.

\section{D numerical simulation of calcium model}

To fulfill the requirement of the finite element method in three dimensional space we consider a tetrahedral mesh of both domain. Figure 8 shows a section of the external domain mesh (Cytosolic domain) and a meshing of the sarcoplasmic domain. The right figure present the section of $\Omega_{s r}$ where the visualization of $\mathrm{Ca}^{2+}$-concentration occur.
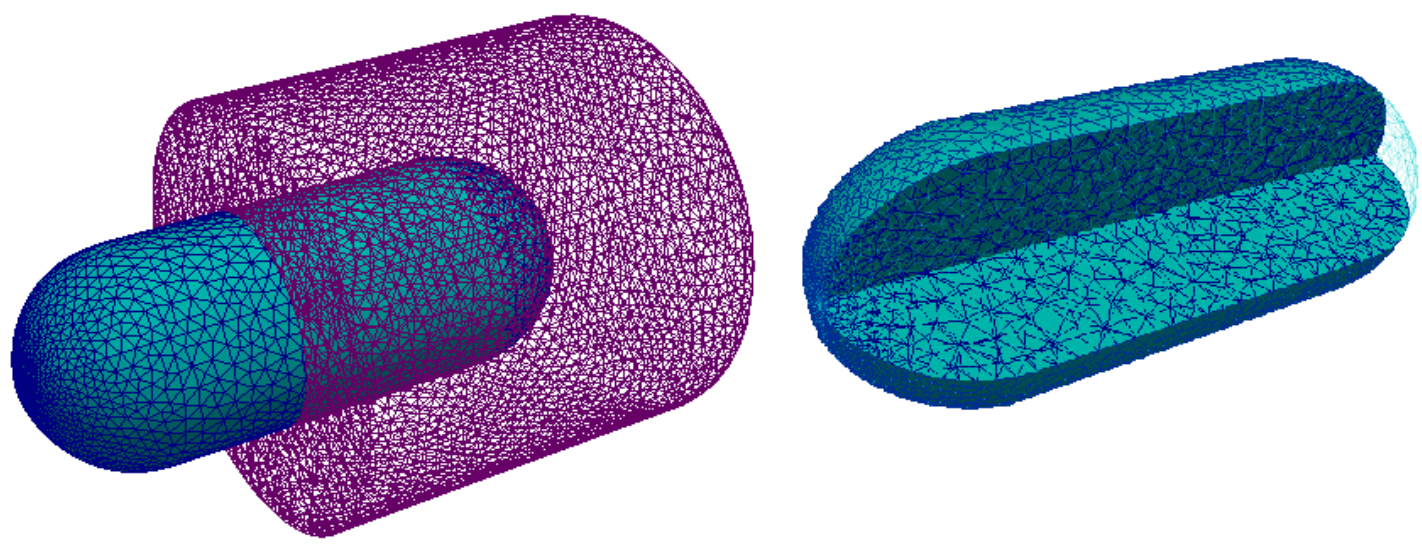

Figure 8. In the left hand-side we show complete mesh grid of $\Omega_{s r}$ and a section of mesh grid of $\Omega_{c y t}$. In the right hand-side we show the volume section in which $3 \mathrm{D}$ simulation will be visualized.

In Figure 9, we present a numerical visualization of calcium solution in both domains. In the sarcoplasmic domain, we show the calcium distribution on the internal sarcoplasmic membrane. We choose to present the calcium dynamics in two different 2D sections with different distance from the ionic channel. To clarify the calcium wave sharpness, we have depict different isosurfaces. Each one describes a specific value of calcium 
concentration. The value of isosurfaces equidistantly varying between 0.14 and 0.44 for linear and nonlinear diffusion cases.

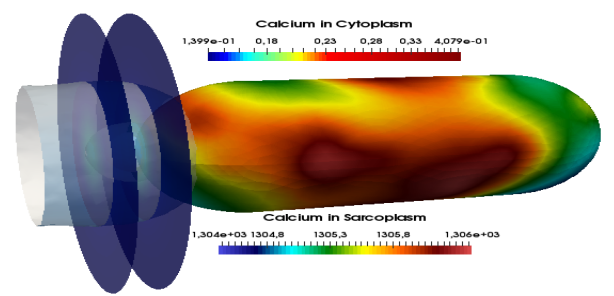

(a) $t=3 \times 10^{-5}$

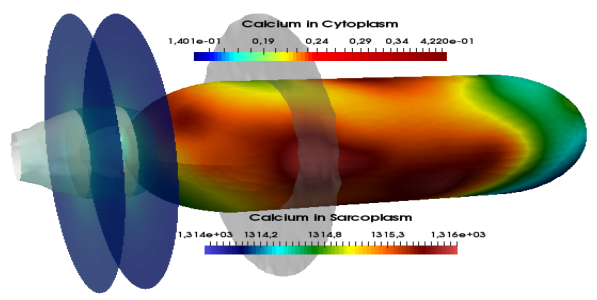

(c) $t=10 \times 10^{-5}$

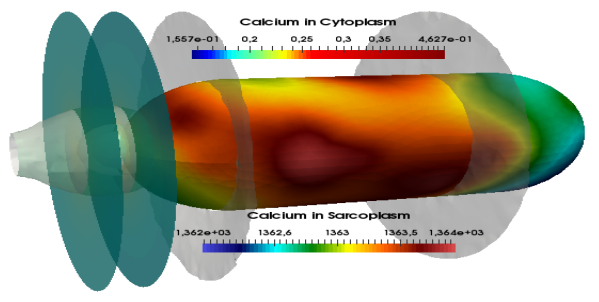

(e) $t=50 \times 10^{-5}$

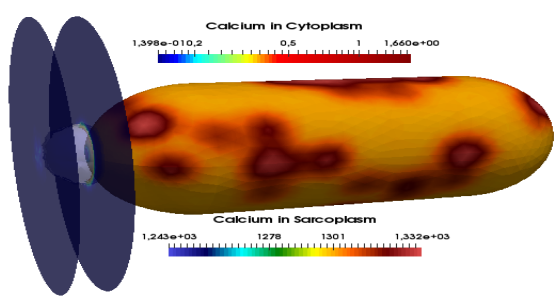

(b) $t=3 \times 10^{-5}$

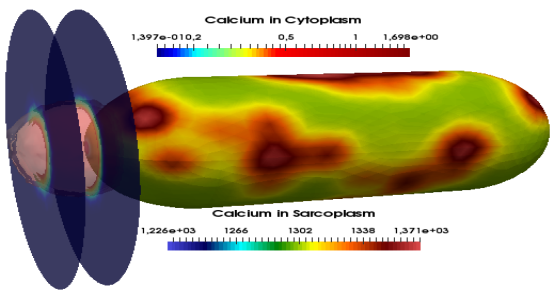

(d) $t=10 \times 10^{-5}$

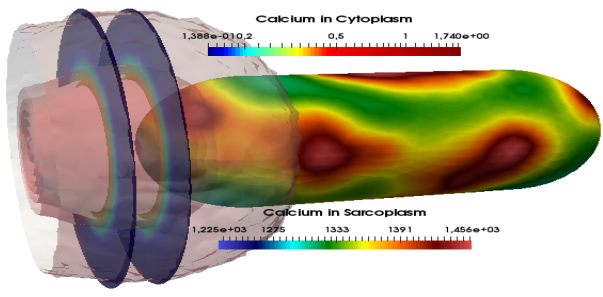

(f) $t=50 \times 10^{-5}$

FiguRE 9. 3D presentation of calcium distribution in both domain.

In Figure 10, we plot the discs shown in Figure 9. The white curves (in Figure 10) present the line curves attached to values of calcium concentrations. 

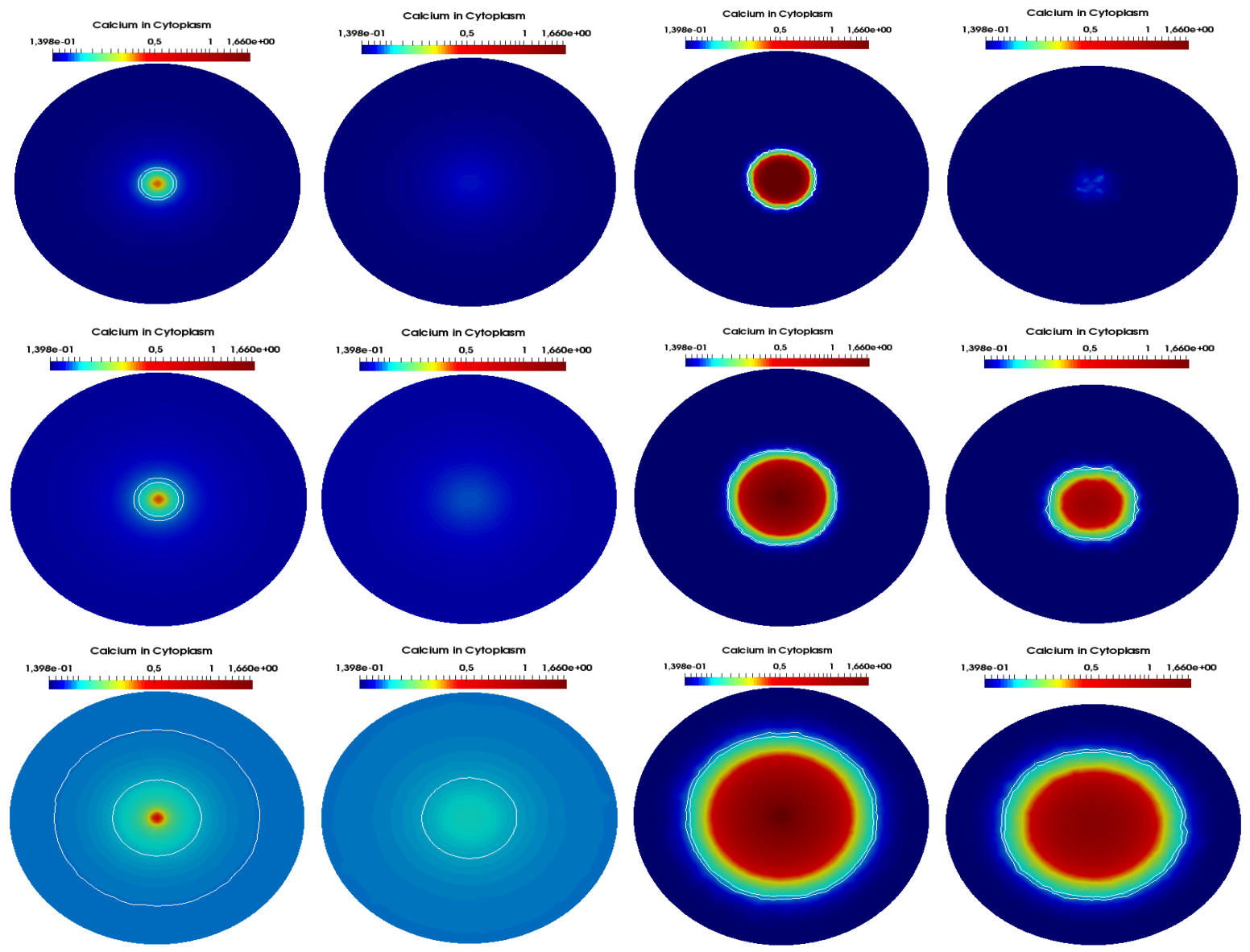

FIGURE 10. The first (resp. second) left column present the linear $\mathrm{Ca}^{2+}$ distribution near (resp. far from) of disc to the ionic channel. The third (resp. forth) column presents the nonlinear distribution of $\mathrm{Ca}^{2+}$ near (resp. far from) of the ionic channel.

To understand the calcium wavefront differences between the anomalous diffusion model and a linear model, we have computed the calcium distribution in both cases in different time frames. Here, we clearly notice the main difference between the linear diffusion and nonlinear diffusion. In Figure 9, the isosurfaces present a splaying dynamics due to the linear diffusion in the medium comparing with the anomalous diffusion. The spatial dynamics in Figure 10 clarifies the anomalous diffusion between the linear model (see for e.g. [5]) and the proposed model. In the linear model we observe that calcium concentration is much higher near to the ionic channels than further (to the ionic channels) which has been shown in [5]. But this doesn't cover the heterogeneity of the cell medium since the constant diffusion based on the assumption that the calcium flux is constantly proportional to the calcium gradient in the whole domain. In the right two columns of Figure 10, we observe that the calcium front-wave is sharper than the linear case. The choice of an increasing function of calcium concentration may explain the slow penetration of calcium through the cellular heterogeneous media (we refer to $[10,29]$ for more details). The complex dense cell structure prevents normal diffusion in $\mathrm{Ca}^{2+}$ low concentration, and the fast diffusion occurs in high concentration region due to high kinetics of $\mathrm{Ca}^{2+}$ particles (for more information see [6] and the references therein), which should form a sharp wavefront pattern. This is approved by experimental micro-imaging technics on calcium diffusion in the cardiac myocyte. 
Finally, to test the numerical scheme convergence, we consider a simple geometry on $\Omega_{s r}=[-2,0] \times[0,1]$ and $\Omega_{c y t}=[0,4] \times[0,1]$. In space triangularization, we consider a uniform mesh grid of length $h=\frac{1}{N}$ with $N$ varying from 5 to 250 , the time step $\delta t=0.05$ and the final time value $T=2$. Table 2 shows errors relative to the exact solutions using $P 1$ and $P 2$ finite element. To simplify, we do not consider the interaction with buffers. We have used as an exact solution (for more information, see [13]) the following function :

$$
\begin{aligned}
& s_{e}(t, x, y)=b_{1} \exp \left(-b_{2} t\right) \sin \left(b_{3}(x+2)\right)+b_{4} x+b_{5}+b_{6} \cosh \left(b_{7} x\right) \cos \left(b_{8} y\right) \\
& c_{e}(t, x, y)=a_{1} \exp \left(-a_{2} t\right) \sin \left(a_{3}(x-4)\right)+a_{4} x+a_{5}+a_{6} \cosh \left(a_{7} x\right) \cos \left(a_{8} y\right)
\end{aligned}
$$

The functions $c_{e}(t, x, y)$ and $s_{e}(t, x, y)$ satisfies the transmission boundary problem for a specific constant. Let $A=100, C=-10, k_{1}=2, k_{2}=2, a_{1}=-310, a_{3}=-0.0072, a_{4}=-1.97$. The other constants are defined as follows :

$a_{8}=\frac{k_{1} \pi}{4}, b_{8}=\frac{k_{1} \pi}{2}, a 7=a_{8}, b_{7}=b_{8}, a_{6}=\frac{A}{\cosh \left(4 a_{7}\right)}, a_{6}=\frac{C}{\cosh \left(2 b_{7}\right)}, a_{2}=k_{s} a_{3}^{2}, b_{2}=a_{2}, b_{3}=\sqrt{\frac{D_{s} a_{3}^{2}}{D_{c}}}, b_{4}=$ $\frac{a_{4} D_{s}}{D_{c}}, b_{1}=\frac{D_{s} a_{1} a_{3} \cos \left(4 a_{3}\right)}{D_{c} b_{3} \cos \left(2 b_{3}\right)}$ and $a_{5}=s_{0}+4 a_{4}, b_{5}=c_{0}-2 b_{4}$ For the conductance value:

$$
g(t, y)=\frac{D_{s} a_{1} a_{3} \exp \left(-a_{2} t\right) \cos \left(4 a_{3}\right)+a_{4}}{b_{1} \exp \left(-b_{2} t\right) \sin \left(-2 b_{3}\right)+b_{5}+b_{6} \cos \left(b_{3} y\right)}
$$

After running the test using different parameters. We present in Table 2 different values of the error

$$
e_{L^{2}}^{2}=\iint_{\Omega_{c y t, T}}\left|c_{e}-c_{h}\right|^{2} d x d t+\iint_{\Omega_{s r, T}}\left|s_{e}-s_{h}\right|^{2} d x d t
$$

and

$e_{H^{1}}^{2}=\iint_{\Omega_{c y t, T}}\left|c_{e}-c_{h}\right|^{2} d x d t+\iint_{\Omega_{c y t}, T}\left|\nabla c_{e}-\nabla c_{h}\right|^{2} d x d t+\iint_{\Omega_{s r, T}}\left|s_{e}-s_{h}\right|^{2} d x d t+\iint_{\Omega_{s r, T}}\left|\nabla s_{e}-\nabla s_{h}\right|^{2} d x d t$.

Hence, we have the following table :

\begin{tabular}{|c|cc|cc|}
\hline & \multicolumn{2}{|c|}{$P_{1}$} & \multicolumn{2}{c|}{$P_{2}$} \\
\hline$N$ & $e_{L^{2}}$ & $e_{H^{1}}$ & $e_{L^{2}}$ & $e_{H^{1}}$ \\
\hline \hline 20 & 0.163607 & 1.20455 & 0.00106206 & 0.0910758 \\
40 & 0.0413642 & 0.307202 & $6.92604 \mathrm{E}-5$ & 0.0121671 \\
60 & 0.0184233 & 0.137055 & $1.3835 \mathrm{E}-5$ & 0.00368335 \\
100 & 0.00663965 & 0.049437 & $1.80705 \mathrm{E}-6$ & 0.000809208 \\
160 & 0.00259459 & 0.0193244 & $2.76497 \mathrm{E}-7$ & 0.000199436 \\
250 & 0.0010629 & 0.00791734 & $5.09595 \mathrm{E}-8$ & $5.25769 \mathrm{E}-5$ \\
\hline
\end{tabular}

TABLE 2. This table represents an estimate of the error between the exact solution and the approximated solution

\section{Conclusion \& Perspective}

In this work, we have proposed a nonlinear reaction diffusion model describing the evolution of calcium in cardiac cell. Based on compactness results, we have studied the well posedness of the problem. Then, we have used a finite element scheme along with implicit Euler discretization to approximate the biochemical substance 
concentrations. We attached a convergence analysis of our scheme using Kolmogorov compactness results. Moreover, we have produced numerical simulations to clarify the physiological link between the given parameters and calcium behavior under linear and anomalous diffusion. On the experimental scale, B. S. Donahue and R. F. Abercrombie [8] deduced that the calcium diffusion in cytosol depends on calcium concentration. Advanced micro-imaging technology in cardiology provides sophisticated results on calcium diffusion profile. Figure 6 proves encouraging similarities between $\mathrm{Ca}^{2+}$-distribution and imaging results shown in [7]. A precise prediction of spacial distribution (including nonlinear diffusion properties) in cytosol is widely important, it may offer some explanation of spontaneous $\mathrm{Ca}^{2+}$-sparks since passive ionic receptor are very sensitive to local calcium concentration. The linear models $[5,11,17,19,24,25]$ has shown some paradoxical results with respect to experiment, our model offers a general framework that may give a step ahead to solve incompatibilities between classical models and experiment. In further work, we intend to simulate the therapeutic strategy by targeting the ionic channel's characteristics and establishing a nonlinear optimal control problem on calcium dynamics.

\section{REFERENCES}

[1] Anaya, V., Bendahmane, M., Mora, D. and Baier, R. R. On a vorticity-based formulation for reaction-diffusion-Brinkman systems. Networks and Heterogeneous Media, 13(1):69-94, 2018.

[2] Bers, D. M., Excitation-contraction coupling and cardiac contractile force. 2nd edition, Dordrecht: Kluwer Academic Publishers, 2001.

[3] Brezis, H., Analyse fonctionnelle, théorie et applications. Masson, Paris, 1983.

[4] Centor, R. M., Allison, J. J., Weissman, N. W., Canto, J., Heudebert, G. R., Juarez, L., and Kiefe, C. I., Diffusion of troponin testing in unstable angina patients: adoption prior to guideline release. Journal of Clinical Epidemiology. 56(12):1236-1243, 2003.

[5] Chai, J., Hake, J., Wu, N., Wen, M., Cai, X., Lines, G. T., Yang, J., Su, H., Zhang, C. and Liao, X., Towards simulation of subcellular calcium dynamics at nanometre resolution. The International Journal of High Performance Computing Applications. 29(1):51-63, 2013.

[6] Crank J., The Mathematics of Diffusion. Brunel University Uxbridge second edition, 1975.

[7] Chen, X., Guo, L., Kang, J., Huo, Y., Wang, S. AND Tan, W. Calcium waves initiating from the anomalous subdiffusive calcium sparks. Journal of The Royal Society Interface, 11(91):20130934, 2013.

[8] Donahue, B. S. and Abercrombie, R., Free diffusion coefficient of ionic calcium in cytoplasm. Cell Calcium, 8(6):437-448, 1987.

[9] Flauraud, E., Nataf, F. and Willien, F., Optimized interface conditions in domain decomposition methods for problems with extreme contrasts in the coefficients. Journal of Computational and Applied Mathematics, 189(1):539-554, (2006).

[10] Gal, N. And Weihs, D. Experimental evidence of strong anomalous diffusion in living cells Physical Review, 81(2), 2010.

[11] Hake, J., Edwards, A. G., Yu, Z., Kekenes-Huskey, P. M., Michailova, A. P., Mccammon, J. A., Holst, M. J., Hoshijima, M. and Mcculloch, A. D., Modelling cardiac calcium sparks in a three-dimensional reconstruction of a calcium release unit. Journal of Physiology, 590(18):4403-22, 2012.

[12] Hurtado, D. E., Castro, S. and Gizzi, A. Computational modeling of non-linear diffusion in cardiac electrophysiology: A novel porous-medium approach Computer Methods in Applied Mechanics and Engineering, 300:70-83, 2016.

[13] Jæger, K. H. An Investigation of Necessary Grid Resolution for Numerical Simulations of Calcium Dynamics in Cardiac Cells, Phd-thesis, 2015.

[14] Kenakin, T. The mass action equation in pharmacology. British Journal of Clinical Pharmacology, 81(1):41-51, 2016.

[15] Ladyzhenskaya, O.A., Solonnikov, V., Uralceva, N., Linear and quasi-linear equations of parabolic type. Transl. AMS 23, Providence, (1968).

[16] Lagnese, J. E. and Leugering, G. Dynamic Domain Decomposition in Approximate and Exact Boundary Control in Problems of Transmission for Wave Equations. Siam Journal on Control and Optimization, 38(2):503-537, (2000).

[17] Langer, G. and Peskoff, A., Calcium Concentration and Movement in the Diadic Cleft Space of the Cardiac Ventricular Cell. Biophysical Journal, 70(3):1169-1182 1996.

[18] Lehnart, S. E., Wehrnes, X. H. T., Kushnir, A. and Marks, A. R., Cardiac Ryanodine Receptor Function and Regulation in Heart Disease. Annals of the New York Academy of Sciences, 1015(1):144-59, 2004.

[19] Louch, W. E., Hake, J., Jølle, G. F., Mørk, H. K., Sjaastad, I., Lines, G. T. and Sejersted, O. M., Control of Ca2+ Release by Action Potential Configuration in Normal and Failing Murine Cardiomyocytes. Biophysical Journal, 99(5): 1377-1386, 2010 .

[20] Marks, A. R., Calcium and the heart : a question of life and death. Journal of Clinical Investigation, 111(5): 597-600, 2003.

[21] Mayer, U. M., Gerstenberger, A. and Wall, W. A., Interface handling for three-dimensional higher-order XFEM-computations in fluidstructure interaction. International Journal for Numerical Methods in Engineering, 79(7):846-869 (2009). 
[22] Nilsson, L. and Stenström, Gas diffusion through sheets of fibrous porous media. Chemical Engineering Science, 50(3):361-371, 1995.

[23] Perin, E. C., Silva, G. V. and Willerson., T. J. An Essential Guide to Cardiac Cell Therapy. No. $1,2006$.

[24] Peskoff, A. and Langer, G., Calcium Concentration and Movement in the Ventricular Cardiac Cell during an ExcitationContraction Cycle. Biophysical Journal, 74(1):153-74, 1998.

[25] Peskoff, A., Posit, J. and Langer, G., sarcolemmal calcium binding sites in heart: II. Mathematical model for diffusion of calcium released from the sarcoplasmic reticulum into the diadic region. Journal of Membrane Biology 129(1):59-69, 1992.

[26] Picht, E., Zima, A. V., Shannon, T. R., Duncan, A. M., Blatter, L. A. and Bers, D. M., Dynamic calcium movement inside cardiac sarcoplasmic reticulum during release. Circulation Research, 108(7): 847-856, 2011.

[27] Pods,, J., Schönke, J. and Bastian, P., Electrodiffusion Models of Neurons and Extracellular Space Using the Poisson-NernstPlanck Equations-Numerical Simulation of the Intra and Extracellular Potential for an Axon Model. Biophysical Journal, 105(1):242-54, 2013.

[28] Rubinstein, I. Electro-Diffusion of ions. 1st edition, Society for Industrial and Applied Mathematics, Philadelphia, Pa, 1990.

[29] Severs, N. J., Bruce A. F., Dupont E., Rothery S. Remodelling of gap junctions and connexin expression in diseased myocardium Cardiovascular research Review, 80(1):9-19, 2008.

[30] Simon, J., Compact sets in the space $L^{p}(0, T ; B)$. Ann. Mat. Pura Appl., (4)146, 65-96, 1987.

[31] Sobie, E. A., Dilly, K. W., Dos Santos Cruz, J., Lederer, W. J. and Saleet Jafri, M., Termination of Cardiac Ca2+ Sparks: An Investigation Mathematical Model of Calcium-Induced Calcium Release. Biophysical Journal, 83(1):59-78, 2002.

[32] Tao, L. and Yong, H., A generalization of discrete Gronwall inequality and its application to weakly singular Volterra integral equation of the second kind. Journal of Mathematical Analysis and Applications, 282(1)56:62, 2003.

[33] Vest, J. A., Wehrnes, X. H., Reiken, S. R., Lehnart, S. E., Dobrev, D., Chandra, P., Danilo, P., Ravens, U., Rosen, M. R., Marks, A. R., Defective cardiac ryanodine receptor regulation during atrial fibrillation. Circulation, 111(26):2025-2032, 2005.

[34] Williams, G., Chikando, A., Tuan, H. M., Sobie, E., Lederer, W. and Jafri, M., Dynamics of Calcium Sparks and Calcium Leak in the Heart. Biophysical Journal, 101(6):1287-96, 2011. 